\title{
A Novel Numerical Method to Predict the Transient Track Geometry and Thermomechanical Effects through in-situ Modification of the Process Parameters in Direct Energy Deposition
}

\author{
T.R. Walker ${ }^{1}$, C.J. Bennett ${ }^{1}$, T.L. Lee ${ }^{2}$ and A.T. Clare ${ }^{3,4}$ \\ ${ }^{1}$ Gas Turbine and Transmissions Research Centre, Faculty of Engineering, University of \\ Nottingham, University Park, Nottingham, NG7 2RD, UK \\ 2 ISIS Facility, Rutherford Appleton Laboratory, Harwell Oxford, Chilton, Didcot, OX11 0QX, \\ $U K$ \\ ${ }^{3}$ Advanced Manufacturing Research Group, Faculty of Engineering, University of \\ Nottingham, University Park, Nottingham, NG7 2RD, UK \\ ${ }^{4}$ Department of Mechanical, Materials and Manufacturing Engineering, Faculty of Science \\ and Engineering, University of Nottingham China, 199 Taikang East Road, University Park, \\ Ningbo 315100, China
}

\begin{abstract}
Direct Energy Deposition (DED) is being widely used to repair damaged components to increase service life and economical operation. Process parameters including laser power, traverse speed and the mass flowrate of the feedstock material may be adapted in-situ. This allows bespoke repair strategies to be devised to match the variability in the condition of the parts supplied that require repair; however, there are limited modelling techniques that allow the adaptive control within the DED process to be represented. In this study, a novel modelling strategy is presented which allows the DED process to be modelled in a transient state. This allows varying process parameters to be included in the model, to predict the transient track geometry and the associated thermomechanical effects of the process. Here, a single-track deposition of IN718 with a varying cross section has been modelled utilising the proposed approach. The modelling methodology was validated with a corresponding experimental study on a deposition made using a Nd:YAG laser source with a coaxial nozzle. An in-situ modification was generated by variation of the laser power profile. The track profile was compared against focus variation microscopy images and the thermomechanical portion of the model was validated through in-situ temperature measurements, micrographs and residual stress, obtained from neutron diffraction measurements. A good agreement between the predicted and experimental findings were observed. The track height and width were predicted with a maximum error of $6.5 \%$ and $7.6 \%$ respectively. The peak temperature and residual stress were predicted within $6.2 \%$ and $11.4 \%$ respectively.
\end{abstract}


Overall, the modelling method presented will allow complex and bespoke multi parameter repair strategies to be rapidly developed.

Keywords: Direct Energy Deposition; Repair; Laser Cladding; Numerical Modelling; Residual Stress

\section{Introduction}

Additive Manufacturing (AM) techniques, such as Direct Energy Deposition (DED), are being used within the advanced manufacturing sector to repair high value components, this removes the need to remanufacture or replace the component, reducing operational costs. Often, DED is utilised over traditional welding techniques due to the high material utilisation ratio, increased accuracy and increased flexibility during manufacture. The nature of the DED process allows for in-situ modifications to the process parameters, which could result in greater control of the track geometry, cooling rates and subsequently the mechanical properties of the component. As not all repair requirements are identical, this type of deposition strategy would inherently allow bespoke and flexible repairs to be implemented. The development of more flexible solutions could also allow the distortion and the evolution of stresses within a component to be controlled during repair, reducing the need for additional processing such as heat treatment or further machining.

The DED process involves a laser source irradiating a metallic substrate to generate a moving melt pool. The feedstock material may be delivered in powder, wire or strip form and conveyed to the work area using a nozzle and an inert gas stream [1]. A moving melt pool is generated from the deposition head moving relative to the substrate surface [2], which, coupled with the incoming material forms a raised track. The entire process is controlled through $\mathrm{CNC}$, therefore in-process modifications can be implemented with ease. When no modification is made to the parameters a steady-state track geometry is formed. Variations in the process parameters will not only lead to a transient geometry being formed, but variations in the temperature fields, which drive the stress condition during solidification, will occur. Therefore, a tool which allows the prediction of the track profile, thermal field and residual stress evolution, due to process parameter variation, would allow bespoke repair procedures to be developed.

Experimental depositions and numerical predictions of a track geometry with an in-situ parameter variation have not been reported to date. Despite this, predictions of track geometries and the thermomechanical effects of the process, utilising different modelling methods, has received attention within the literature. Early models to determine track profiles were derived empirically across a range of process parameters, often neglecting the physical complexities of the process such as the interaction of the powder feed distribution 
and the melt pool [3]. An empirical model, presented by Kumar et al. [4], allowed the track height to be derived based on the laser power and the material mass flow rate. Although the time required to predict the track height was minimal, experimental data was required to calibrate the model. This was required to reduce the maximum error between the predicted and experimental height from $50 \%$ to $13 \%$. To increase the accuracy of the approach, an empirical statistical model was later used to include more parameters of the process. Davim et al. [5], utilised a similar method, with the final equation being derived using a Multiple Regression Analysis (MRA) model. The track height and width were determined with an error of $7.6 \%$ and $6 \%$ respectively. Despite the volume of data collected to inform the model, the percentage error for the predicted melt pool depth was $20.1 \%$ showing a level of inaccuracy with this approach. The main issue with using empirical/statistical models is the experimental data needed to derive the model. This would not be feasible to design repair strategies as each repair would be bespoke. In addition to this, the empirical approach is limited in providing accurate predictions within the bounds of the process parameters used to define the model. Therefore to enable the effects of in-situ modification to be evaluated for bespoke repair applications, a high number of experiments would be required to enable the correct repair scheme to be designed. As this would be an inefficient approach, leading to a lengthy and costly design process, a generalised modelling method which allows the process to be simulated is therefore required.

Analytical models have recently been used to allow for a more flexible modelling approach, as the methodology is no longer bound by a range of process parameters. An early model presented by Picasso et al. [6] determines the parameters required to fabricate a deposition of a prescribed height through solving a series of analytical expressions. An iterative solution procedure was utilised to reassess the thermal field through recalculation of the power absorbed by the workpiece. The power loss and interaction between the laser beam, substrate and powder particles were considered at each iteration. The solution procedure was terminated when the relative change between the absorbed power, laser velocity and the powder mass flow rate was less than a prescribed value. A major drawback of this approach was that the track height had to be predefined, therefore, no assessment could be made on how the process parameters affected the deposition geometry. A simplified approach presented by Pinkerton and Li [7], modelled the geometry of a moving melt pool and the subsequent deposition track through an energy and mass balance. The laser process was simplified by assuming that the heat source and track profile could be represented as a point source and a circular arc respectively. The approach was relatively simple and was not computationally demanding, therefore providing a quick prediction of the track geometry. The model was used to assess the effect of the laser power on the steadystate track width and height, with a maximum error of $11.1 \%$ and $38.9 \%$ respectively; therefore, the validity of the approach has to be assessed when analysing the effects of the process parameters on the deposition geometry. A hybrid model of a numerical and analytical approach was presented by Ahsan and Pinkerton [8], which determined the track 
height, utilising a fully-coupled mass-enthalpy balance. Similar to Picasso et al. [6], the thermal field was reassessed to include the power losses associated with the process. An analytical temperature field, solved through numerical integration, was used to determine the melt pool geometry. The track profile was calculated from analysing the interaction between an analytical representation of the powder flux distribution and the predicted melt pool geometry. The model was verified experimentally, with a maximum error of $8.4 \%, 3 \%$ and $20 \%$ for the peak temperature, track width and height respectively. Through representing the key physics of the process, a more accurate representation of the track profile was determined. For most analytical models, the time required to determine a solution is minimal, as the models are not computationally demanding as a discretised domain is not required. Therefore, when designing bespoke repair strategies and evaluating the effects of the process parameters on the track geometry, these models are beneficial. However, as a quasi-static solution of the thermal field is calculated, the time dependent history of the process is neglected; therefore, the effect of in-situ parameter modifications cannot be evaluated using analytical models. Also, when determining an analytical thermal field, the geometry is often simplified to an infinite plate. Therefore, no real assessment of the repair strategy can be assessed with these methods using the true component geometry. It should be noted that analytical modelling to predict residual stresses are very limited for DED; however, a model presented by Tamanna et al. [9] predicted the residual stress for laser cladding using a one-dimensional model. The model was used to evaluate the effects of preheating the substrate at different temperatures on the final residual stress field. No experimental validation of the proposed methodology was completed and only a onedimensional representation of the stress field could be predicted. Therefore, evaluation of the thermomechanical effects for the entire component would not be possible utilising this approach.

Numerical models of the DED process tend to focus on calculating the thermal histories and thermal distributions of the final part. In some cases these types of models are used to predict track geometries and can be extended to predict residual stress fields. Early numerical models applied the heat flux to an unchanging surface, however, the inclusion of material deposition can be incorporated through element manipulation techniques [10]. Numerical models using computational fluid dynamics (CFD) have been used to predict the physical phenomena from the deposition head to the melt pool dynamics, with some of these models allowing the formation of the track to be included [11], [12]. However, as CFD cannot be used to predict the thermomechanical effects of the process, a review of these methodologies will not be included. Therefore, the focus will be on the finite element method, specifically to predict the track geometry and the thermomechanical effects of the process. A three dimensional model for DED, utilising both an analytical and numerical approach was derived by Labudovic and Kovacevic [13]. An analytical model based on Green's function was used to determine the thermal field of the process and this was compared to the FE simulation. A numerical model to determine the residual stress field for a wall structure was 
also presented, in an attempt to determine a feedback control to reduce residual stresses during the deposition. Experimental data for both the thermal and mechanical portions of the model were used to validate the approach, with the use of a high speed camera and x-ray diffraction data. Although a good agreement was present between the experimental data and the modelling method, a square track geometry was used for the FE model which does not reflect the true track geometry. The interaction between the powder feed and melt pool were not included; therefore, a prediction of the track profile was not possible. Utilising this methodology, a bespoke repair solution could not be designed, as a true representation of the track geometry was not included in the model; also, as the powder feed was not included, in-situ variation of the process parameters could not be incorporated with this approach. A model was presented by Farahmand and Kovacevic [14] which included a more accurate representation of the track geometry and was used to predict the thermomechanical effects of high power direct laser deposition. The track geometry was not determined through a numerical method and was implemented into the model through geometrical measurements of the experimental track. A good agreement was attained between the experimental and predicted melt pool geometry with a maximum error of 3.8\%; therefore, validating the approach used to determine the melt pool geometry. The residual stresses were determined for a planar section and compared to x-ray diffraction results. A maximum error of $10 \%$ was reported for the peak stress. Although the presented methodology for the thermomechanical portion of the model was accurate when compared to experimental data, the flexibility of the approach was reduced as no track profile could be determined without prior measurement of the experimental geometry. Peyre et al. [1] proposed a three step numerical-analytical model to predict the track geometry and thermal loadings induced by the process. The first step required calculation of the temperature profile in the powder stream, giving a factor of 2 overestimation. This was used as a boundary condition for the thermal model. A fixed laser heat source with a quasi-spherical formulation was used. The track height was predicted by discretising the melt pool further and analysing the total element contribution to the layer growth. The track width and height were predicted to within $10 \%$ and $13 \%$ of the experimental findings. No track profile was derived from the model, with the track being represented as square elements in the subsequent thermal models. As no track geometry was determined, the method would not allow a true representation of the repair scheme to be modelled. It should also be noted that as no moving heat source was used within the thermal model, a transient history of the process could not be predicted, therefore neglecting the flexibility in repair solutions that could be obtainable. A more recent study presented by Zhang and Kovacevic [15], determines the thermomechanical effect of DED using an uncoupled FE model to simulate cladding of dissimilar materials. The interaction between the laser and powder was introduced by altering the power attenuation. Element birth and death was utilised to include the effect of material addition during the process; however, the cross-section of the track was uniform and determined through experimental measurements. Validation of the thermal model was completed through thermocouple 
measurements and the maximum error between the peak temperatures was $5.8 \%$. No experimental validation for the mechanical model was presented as part of this study. Although a better representation of the process was conducted using this model, the track geometry could not be implemented into the FE model until an experimental deposition has been completed; therefore, reducing the flexibility of the modelling approach. Walker et al. [16] presented a 3D analytical-numerical model of the deposition process to predict the thermomechanical effects induced from a single-track deposition. A mass-enthalpy balance was used to predict the track geometry from an analytical powder distribution, with the melt pool geometry being determined from an analytical temperature field. The track width and height were predicted within $1.98 \%$ and $0.43 \%$ of the experimental track respectively. Implementation of the predicted track geometry, within the thermomechanical analysis, gave a maximum error for the peak temperature and residual stress of 3.1\% and 19\% respectively. Although an accurate modelling strategy was presented for the deposition process, the model could not predict geometries or thermomechanical fields that contained an in-situ parameter variation. A more advanced model presented by Alimardani et al. [17] determined the formation of a 3D track geometry for the entire deposition process, utilising a constant set of process parameters. A 3D numerical heat transfer model was utilised to determine the boundary of a moving melt pool. At each time step of the analysis, the mesh was altered to introduce the addition of material to the domain. The height and width of the track were determined from the powder feed rate, elapsed time and the interaction between the melt pool and powder stream. A standard objective function in the form of an implicit second order polynomial was used to represent the track geometry; therefore, an idealised representation of the geometry was used for the model. The model allowed a transient representation of the track profile to be determined and the subsequent thermal fields during the process. The peak temperatures were overestimated by the model with a maximum error of 8\%. Experimental validation of the average track height was completed with a maximum error of $19.35 \%$ for single track geometries. No attempt was made to determine the residual stress fields based on the derived thermal field. It should be noted that although the track formation could be determined over a defined time period, no attempt was made to investigate in-situ parameter variation on the track geometry.

The current uptake of in-situ parameter variation for repair processes is restricted by the modelling approaches not incorporating these phenomena; therefore, the only method to quantify the effect of process variations would be through the use of costly experimental trials. Although methodologies exist to predict the track formation within DED, using transient formulations or steady-state equivalents, these approaches are limited to a constant set of process parameters; therefore, to allow the inherent manufacturing flexibility of the DED process to be encapsulated in a modelling approach, a method which can incorporate variation of the process parameters during the deposition is required. In response, this study presents a generalised modelling strategy which predicts a 3D transient track profile determined from in-situ variations of the process parameters. Through the use 
of an analytical and FE numerical model, the track formation was predicted by considering the interaction of the powder flow distribution with the melt pool geometry. This was solved using a fully coupled mass and enthalpy balance to calculate the associated power losses through assimilation of the powder. To allow the thermal and mechanical effects of the deposition strategy to be predicted, a sequentially coupled thermomechanical analysis was implemented. To validate the proposed methodology, at each stage of the modelling process, an experimental case study is presented. With the use of the FE method for the proposed modelling strategy, an assessment of the repair methodology can be completed for the true component geometry. The developed method will therefore allow for bespoke repair strategies to be designed, without the need for lengthy and costly experimental trials. Complex parametric studies during the design process can be conducted using the presented methodology allowing an assessment of the proposed repair strategy on the distortion and residual stress formation within the repaired component to be completed. As the model predicts deposition tracks with varying cross sections, an optimum tool path can also be determined to ensure a more efficient use of the deposition material by only refilling the damaged area of the component. As a more tailored repair solution can be determined using the proposed tool, a reduction in the secondary processing of the repaired component may be possible, which is advantageous for reducing repair costs.

\section{Methodology}

The DED process allows for in-situ variation to process parameters during the deposition of a track. As the heat source, powder feed and traverse table are all coupled with a CNC system, effectively near infinite combinations of process parameters are achievable. In-situ modification of the parameters results in the process being in a transient state. When a constant set of parameters is utilised, a uniform track is produced which can be referred to as the steady-state region.

A novel methodology is presented in this work which allows a transient track profile to be determined based on the physics of the process. The approach was then extended to determine the thermomechanical effects through the use of a sequentially-coupled finite element analysis. To validate each stage of the model, an experimental case study is presented in which a deposition, with a varying cross section, is fabricated through an insitu modification to the laser power. The predicted track profile was compared to focus variation microscopy images of the experimentally deposited track. The thermomechanical section of the model was validated through in-situ temperature measurements, metallographic examination of the fusion zone and residual stress, determined using neutron diffraction (ND) measurements.

\subsection{Numerical Modelling Strategy}


The modelling strategy presented in this study incorporates the key process variables in order to model the DED process as a single entity. A flowchart of the modelling strategy used in this study is presented in Figure 1. Firstly, the model predicted the transient track geometry utilising a FE heat transfer model to calculate the evolution of the melt pool geometry. This enabled a $2 \mathrm{D}$ track profile to be predicted at each time step $\left(t_{n}\right)$ of the analysis. The predicted track geometry was then implemented into a sequentially-coupled thermomechanical FE analysis, allowing the thermal and mechanical effects induced by the process to be predicted. All of the FE models in this study were solved using the commercial code ABAQUS.

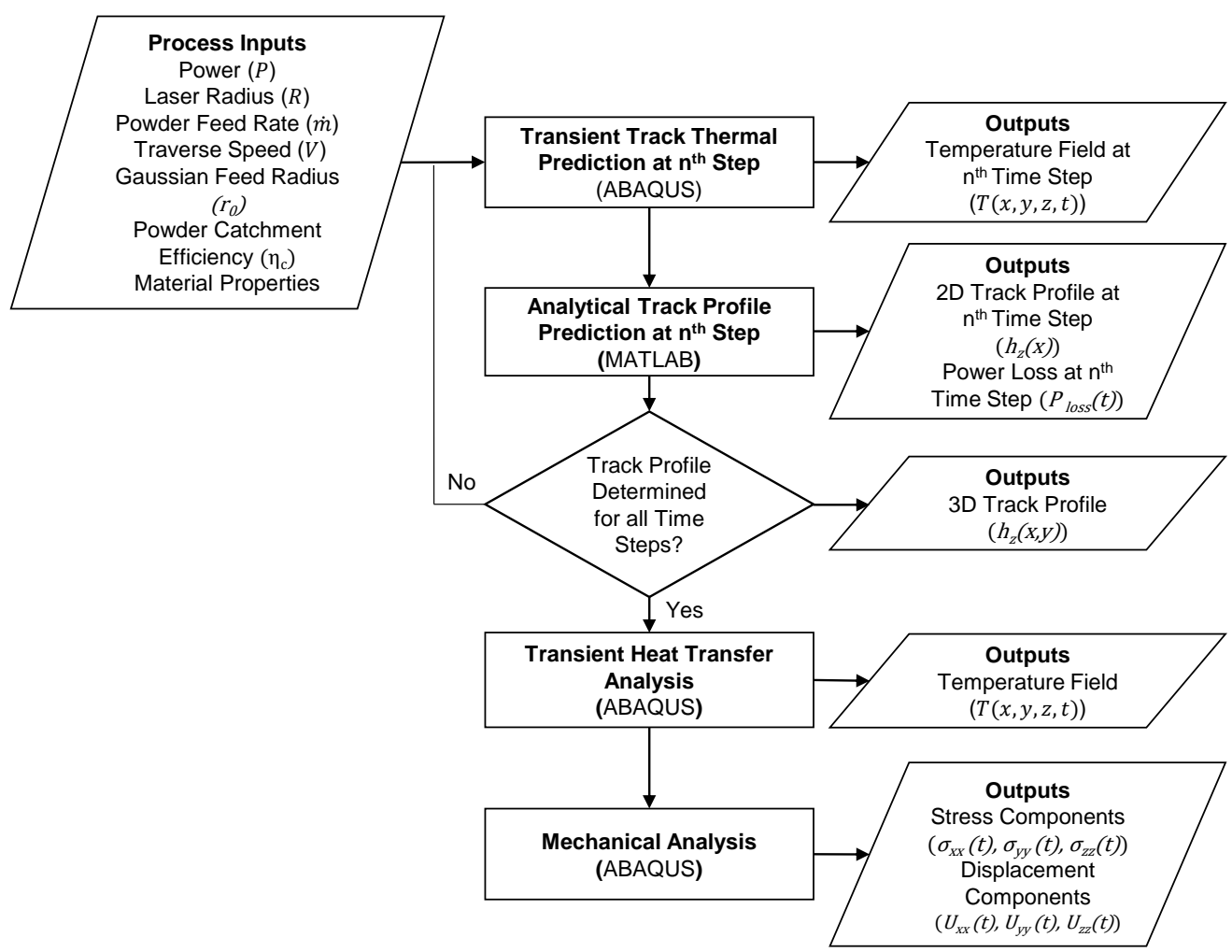

Figure 1 Flowchart of the modelling strategy used to predict the track profile, thermal histories and residual stress fields. The associated inputs and corresponding outputs are shown at each stage of the modelling process.

\subsubsection{Track Profile Model}

Through the adaptive control of the process parameters, a variation in the track geometry will be present and the thermal field will be in a transient state. To allow this phenomena to be represented, a transient FE heat transfer analysis, coupled with an analytical model was utilised. The process parameters, material properties and the meshed FE domain were specified manually with each subsequent step of the analysis being automated. The flowchart of the modelling process is presented in Figure 2. 
To determine the thermal field during the deposition, the transient heat conduction equation was solved, which has the form:

$$
\rho C_{p} \frac{\partial T}{\partial t}(x, y, z, t)=-\nabla \cdot \vec{q}(x, y, z, t)+Q(x, y, z, t)
$$

where $\rho$ is the material density, $C_{p}$ is the specific heat capacity, $T$ is the temperature at time $t, Q$ is the internal heat generation rate, $\nabla$ is the spatial gradient operator and $\vec{q}$ is the heat flux vector. The process parameters were input as a function of time $(P(t), V(t)$ and $\dot{m}(t))$ and were used to determine the track profile at each time step of the model. The heat flux was implemented into the FE model through the user subroutine DFLUX, utilising the circular disc heat source model presented by Pavelic [18]:

$$
Q(x, y, z, t)=\frac{\alpha P(t)-\left(P_{m}+P_{\text {evap }}\right)}{\pi r_{0}^{2}} \exp \left[-\frac{x^{2}+(y-V(t) t)^{2}}{r_{0}^{2}}\right]
$$

where $\alpha$ is the material absorptivity, which was not considered to be temperature dependent, $P(t)$ and $V(t)$ are the laser power and traverse velocity expressed as a function of time, $P_{m}$ is the power required to sustain mass addition, $P_{\text {evap }}$ is the evaporation power loss at the melt pool, $x, y$ and $z$ are the Cartesian coordinates and $r_{0}$ is the Gaussian radius. To be consistent with the analytical approach, convective and radiative heat losses were neglected. Before the track profile could be predicted, an initial heat transfer model was used to determine the time required $\left(t^{\prime}\right)$ for the melt pool to reach a steady-state during the early time steps of the analysis. This time was then used to determine the starting location for which the first track profile was predicted. A schematic of the analysis stages and subsequent outputs are presented in Figure 3.

At each time step $\left(t_{n}\right)$, a 2D track profile was predicted. The melt pool width and length were determined from the nodal FE temperatures. As only a $2 \mathrm{D}$ representation of the melt pool geometry was required, the through thickness nodes were neglected from the postprocessing stage. The melt pool geometry was represented using front and rear elliptical sections [8]:

$$
\begin{aligned}
& y_{f}=L_{n}^{f} \sqrt{1-\frac{4 x^{2}}{W_{n}^{2}}} \\
& y_{r}=L_{n}^{r} \sqrt{1-\frac{4 x^{2}}{W_{n}^{2}}}
\end{aligned}
$$

where: 


$$
\begin{gathered}
T\left( \pm \frac{W_{n}}{2}, 0,0\right)=T_{m} \\
T\left(0, L_{n}^{f}, 0\right)=T_{m} \\
T\left(0, L_{n}^{r}, 0\right)=T_{m}
\end{gathered}
$$

where $W_{n}, L_{n}^{f}$ and $L_{n}^{r}$ are the width, front length and rear length of the melt pool at the current time step. 


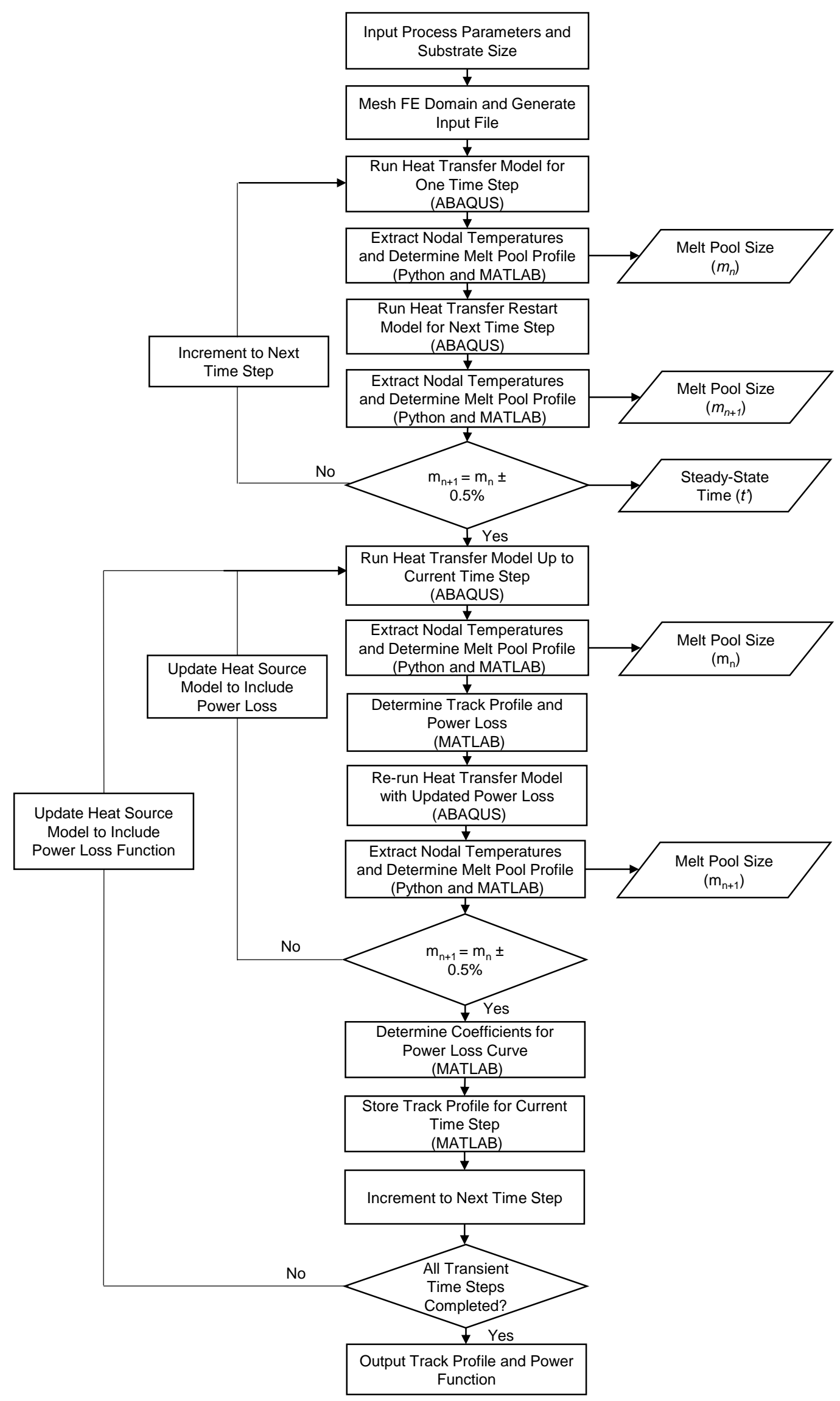

Figure 2 Flowchart of the modelling procedure used to determine the transient track geometry. 


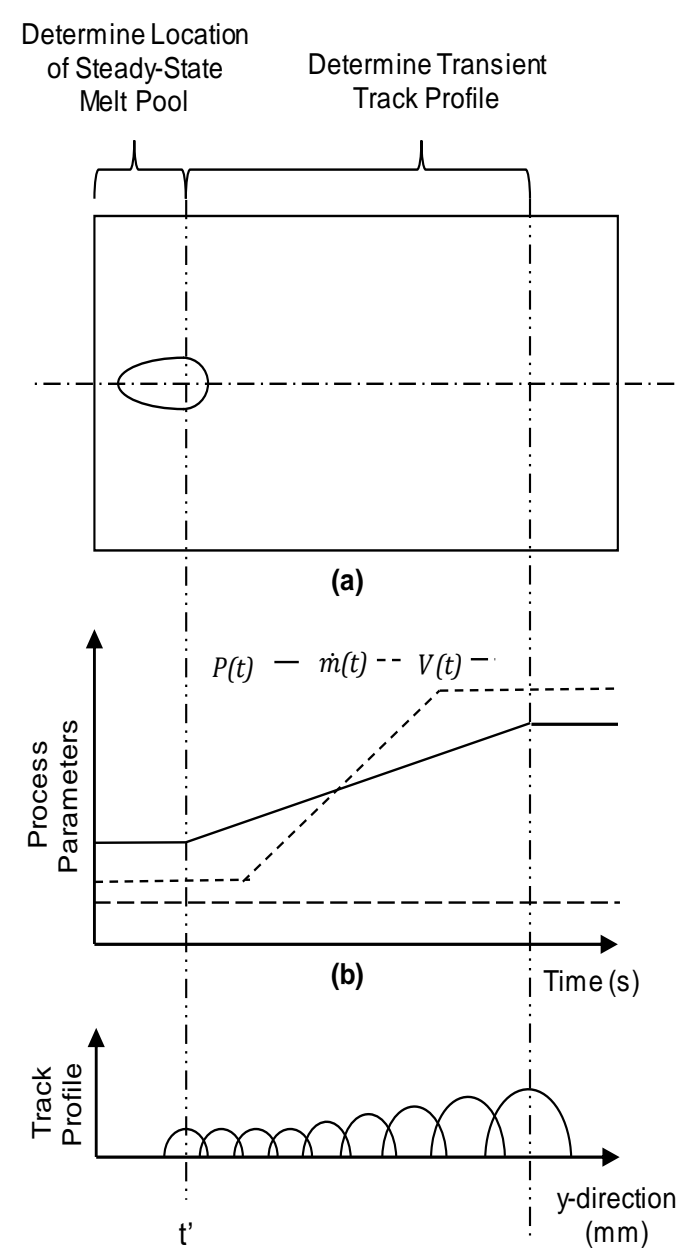

(c)

Figure 3 Schematic of the methodology used to determine a transient track profile (a) shows the substrate geometry identifying the two regions of the modelling process; (b) presents the concept of varying process parameters during the deposition process;(c) schematic of the calculated track profile at each time increment.

The track profile $\left(h_{z}(x)\right)$ was predicted at each time step by integrating a Gaussian surface representing the powder flux distribution, between the melt pool limits:

$$
\begin{gathered}
h_{z}(x)=\frac{\eta_{c}\left(t_{n}\right)}{\rho V\left(t_{n}\right)} \int_{y_{r}}^{y_{f}} q_{p f} d y \\
q_{p f}=\frac{2 \dot{m}\left(t_{n}\right)}{\pi r_{p}^{2}} \exp \left[-2 \frac{x^{2}+y^{2}}{r_{p}^{2}}\right]
\end{gathered}
$$

where $\eta_{c}$ is the powder catchment efficiency, $V\left(t_{n}\right)$ and $\dot{m}\left(t_{n}\right)$ are the traverse velocity and the powder mass flow rate at the current time step and $r_{p}$ is the Gaussian feed radius. The Gaussian feed radius was determined from the powder concentration distribution, where the peak value was equal to [19]: 


$$
C^{\prime}=\frac{2\left[r_{i}+r_{0}\right] \dot{m}\left(t_{n}\right)}{\sqrt{\pi} 0.843 \dot{V} z_{s} \tan (\theta)}
$$

where $r_{i}$ and $r_{0}$ are the inner and outer radius of the nozzle respectively, $\dot{V}$ is the volumetric flow rate of the carrier gas, $z_{s}$ is the stand-off distance and $\theta$ is the initial powder stream angle. It can be assumed that the powder distribution has a Gaussian profile across the stream width [19]. Therefore, the final form of the powder concentration distribution was as follows:

$$
C(x, y, z)=C^{\prime} \exp \left[\frac{x^{2}+y^{2}}{w^{2}}\right]
$$

where $w$ is the width of the powder stream equating [19]:

$$
w=\frac{2\left(r_{0}-r_{i}\right) \tan (\theta)}{\left(r_{0}+r_{i}\right)} z_{S}
$$

The Gaussian feed radius equates the radial position where the powder concentration (eq.7) falls to $e^{-2}$ of the peak value (eq.6). To quantify the powder catchment efficiency, an analytical model was used to represent the powder flow from the coaxial nozzle. The analytical model takes into consideration the geometry of the nozzle, the stand-off distance and the powder flow characteristics at the nozzle outlet. The current model presented by Ahsan and Pinkerton [8] assumes that all the powder is assimilated by the melt pool, however a more realistic approach was implemented here. The realistic approach included the effect of the stand-off distance, melt pool geometry and the powder flow characteristics on the catchment efficiency. The analytical value was modified using the following expression [13]:

$$
\begin{aligned}
& \eta_{m}=\int_{-\frac{W_{n}}{2}}^{\frac{W_{n}}{2}} \int_{y_{r}}^{y_{f}} S(x, y) d y d x \\
& +\int_{-\infty}^{0} \int_{0}^{\sqrt{\left(\frac{W_{n}}{2}\right)^{2}-\frac{X_{\min }^{2}}{4}}} S(x, y) d y d x \\
& -\left[\int_{y_{r}}^{0} \int_{0}^{\frac{W}{2} \sqrt{1-\frac{x^{2}}{y_{r}^{2}}}} S(x, y) d y d x-\int_{\frac{y_{r} X_{\min }}{W_{n}}}^{0} \int_{\sqrt{\left(\frac{W_{n}}{2}\right)^{2}-\frac{X_{\min }^{2}}{2}}}^{\frac{1-\frac{x^{2}}{y_{r}^{2}}}{W^{2}}} S(x, y) d y d x\right]
\end{aligned}
$$

where $S(x, y)$ and $X_{\min }$ are equal to [20]: 


$$
\begin{gathered}
S(x, y)=\frac{1}{2 \pi z_{s}^{2} \tan (\phi)[\cot (\zeta-\phi)-\cot (\alpha)]} \exp \left[-\frac{1}{2}\left[\frac{x^{2}}{\left(z_{s} \tan (\phi)\right)^{2}}\right.\right. \\
\left.\left.+\frac{y^{2}}{\left(z_{s} \cot (\zeta-\phi)-z_{s} \cot (\zeta)\right)}\right]\right] \\
X_{\text {min }}=\frac{\pi D_{\text {particle }} \rho_{\text {powder }} C_{p}\left(T_{m}-T_{\text {amb }}\right)\left(2 D_{\text {laser }}\right)^{2} v_{\text {powder }}}{6 \alpha_{\text {powder }} P(t)}
\end{gathered}
$$

where $\zeta$ is the inclination angle between the nozzle and the substrate, $\phi$ is the divergence angle of the powder stream, $D_{\text {particle }}$ is the diameter of the powder, $\rho_{\text {powder }}$ is the density of the powder, $D_{\text {laser }}$ is the spot diameter of the laser, $\alpha_{\text {powder }}$ is the absorptivity of the powder and the velocity of the powder, $v_{\text {powder }}$, was given by [21]:

$$
v_{\text {powder }}=\frac{4 \dot{V}}{\pi\left(d_{0}^{2}-d_{i}^{2}\right)}
$$

where $d_{0}$ and $d_{i}$ are the outer and inner diameter of the nozzle respectively. The final catchment efficiency was therefore given by [16]:

$$
\eta_{c}\left(t_{n}\right)=\frac{\int_{-\infty}^{\infty} \int_{\infty}^{\infty} q_{p f} d y d x}{\int_{-\frac{W_{n}}{2}}^{\frac{W_{n}}{2}} \int_{y_{r}}^{y_{f}} q_{p f} d y d x} \eta_{m}
$$

As the computational deficit of the modelling approach was through determining the thermal field through a FE analysis, an efficient method to solve the analytical equations for each iteration was required; therefore, the thermal properties were assumed to be temperature independent when solving for the track profile, as this increased the convergence rate for each iteration [8] and in turn, the global computational time was reduced. When implementing eq.4-14, material properties were defined at a temperature halfway between the melting and ambient temperature $\left(20^{\circ} \mathrm{C}\right)$ of the substrate based on the assumptions outlined in [8].

Two sources of power loss were accounted for; the energy required to melt the powder particles $\left(P_{m}\right)[8]$ and the evaporation losses within the melt pool $\left(P_{\text {evap }}\right)$ [22]. These losses were calculated at each time step of the analysis. The total power loss $\left(P_{\text {loss }}\right)$ in the system was therefore equal to:

$$
\begin{gathered}
P_{\text {loss }}=P_{m}+P_{\text {evap }} \\
P_{m}=\rho V\left(t_{n}\right)\left[C_{p}\left(T_{m}-T_{a m b}\right)+L_{m}\right] \int_{-\frac{W}{2}}^{\frac{W}{2}} h_{z}(x) d x
\end{gathered}
$$




$$
P_{\text {evap }}=\frac{\pi}{4} W_{n}\left(L_{n}^{f}+L_{n}^{r}\right)\left(\frac{455.32 \exp \left[4.834-\left(\frac{18836}{T_{\text {mean }}}\right)\right]}{\sqrt{T_{\text {mean }}}}\right) h_{v}
$$

where $L_{m}$ is the latent heat of melting, $T_{\text {mean }}$ is the mean temperature in the melt pool and $h_{v}$ is the latent heat of vaporisation. To allow the effective power to be defined at each time step in the transient region, the power loss was expressed as a function of time $\left(P_{\text {loss }}(t)\right)$. The heat source model was then updated to include this function. A schematic of the power loss history and how it was represented in the model is shown in Figure 4.

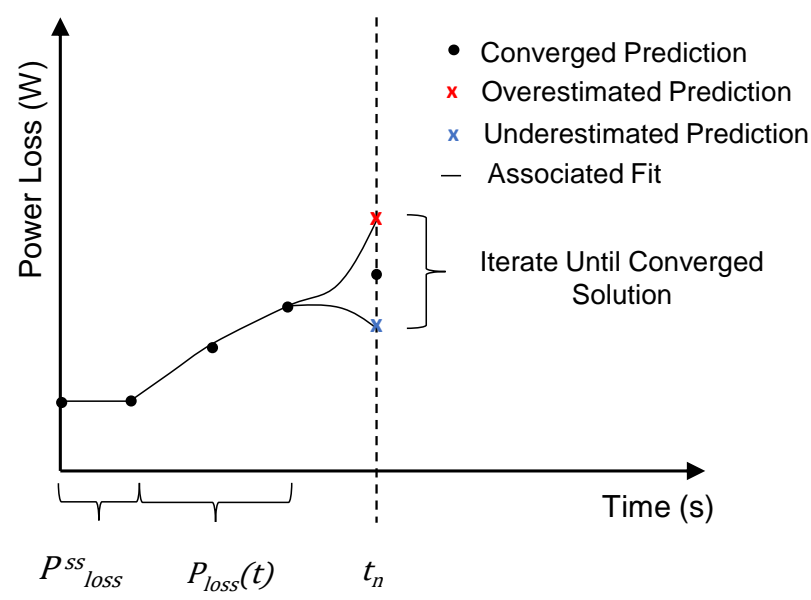

Figure 4 Schematic of the power loss function showing the three possible outputs of the model after an iteration during the current time step.

The power loss was calculated using an iterative solution procedure with the initial guess equating the power loss for the previous time step of the analysis. When the model was not in a transient state, the power loss was assumed to be constant $\left(P_{\text {loss }}^{s s}\right)$ and was determined from Eq.(6). For the first iteration, at the $\mathrm{n}^{\text {th }}$ time step, the model overestimated the power loss and on the second iteration the losses were underestimated [8]. This process continued until a stable melt pool was achieved and the power loss converged. To enable the predicted and converged values to be accurately inputted into the FE heat source model, the selected function had to be able to represent all three possible states of power loss that could be encountered, i.e. overestimate, underestimate and converged solution; therefore, a third order polynomial was selected of the following form:

$$
P_{\text {loss }}(t)=a t^{3}+b t^{2}+c t+d
$$

where $a, b, c$ and $d$ are the coefficients of the equation. The overall solution procedure was iterative to ensure that a stable enthalpy balance and melt pool geometry were achieved at each time step. A stable melt pool occurred when the following criteria was valid: 


$$
\begin{aligned}
0.05 W_{n-1} & \leq W_{n} \leq 0.05 W_{n-1} \\
0.05 L_{n-1} & \leq L_{n} \leq 0.05 L_{n-1}
\end{aligned}
$$

where $W_{n}$ is the melt pool width and $L_{n}=L_{n}^{f}+L_{n}^{r}$ for the current time step of the analysis. Upon a stable melt pool being determined, the power loss and the track profile for each time step were stored. The model was terminated when all the predefined time steps were completed. Each 2D track profile was concatenated to determine the final 3D track geometry over the transient region of the model.

\subsubsection{Thermomechanical Modelling}

To determine the thermal histories and residual stress fields induced from a transient deposition, a sequentially coupled thermomechanical analysis was implemented. The track geometry obtained from section 2.1.1 was imported into the FE domain; a schematic of the process and the adopted modelling strategy is presented in Figure 5.

A transient heat transfer analysis was used to solve the governing equation outlined in Eq.1. The FE model was solved using the full Newton-Raphson integration scheme, with an integration time step equal to moving one element length per step. This was adopted for the heating steps of the analysis only. The integration time was set to automatic for the cooling phase. To simulate the deposition of material, an active/inactive element approach was implemented in both the thermal and mechanical FE model (Figure 5) [10] [23]. The reactivated elements had a predefined temperature equal to the melting point of the material. Thermal effects, due to solidification of the melt pool, were included in the model. To ensure that the heat loss was modelled sufficiently, both convective and radiative losses were accounted for using the Newton and Stefan-Boltzmann laws respectively. As varying portions of the substrate surface were covered during the analysis, the convection and radiative losses were defined only on the free surfaces exposed to the external surroundings. To model the conduction arising between the substrate and the machine bed, an artificial convective boundary condition was applied to the surface in contact. The sink temperature for all the defined losses and the initial substrate temperature were set to $20^{\circ} \mathrm{C}$. 


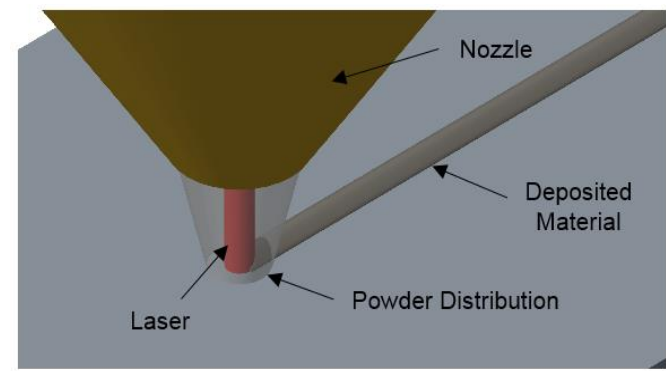

(a)

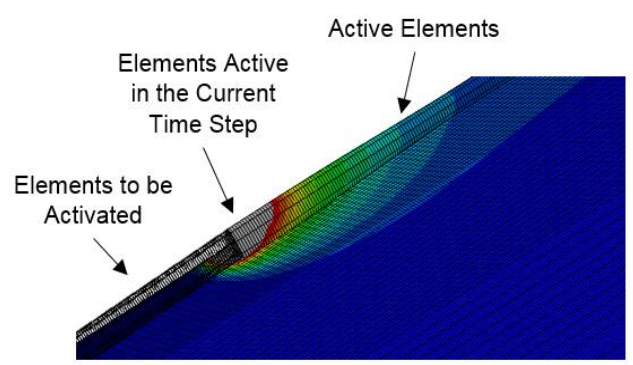

(b)

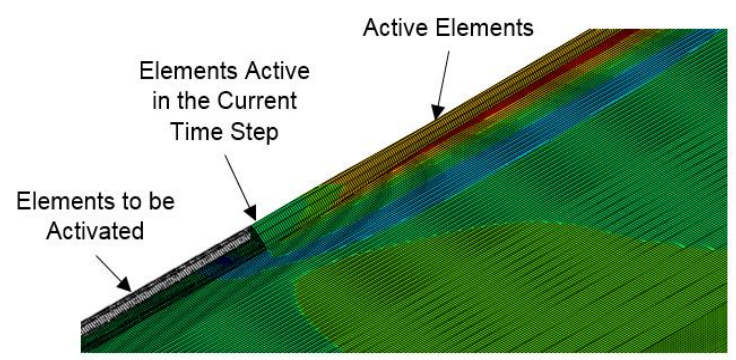

(c)

Figure 5 Schematic of the thermomechanical modelling process for DED (a) realistic representation of the DED manufacturing process; (b) thermal model showing the temperature contour with the adopted element activation strategy; (c) mechanical model showing the longitudinal stress distribution and the adopted element activation strategy.

The FE mesh utilised in the thermal model was also used in the mechanical analysis to allow the thermal field to be transferred to the model correctly. For all of the numerical models, it was assumed that the residual stress formation was driven predominately through thermal effects. Therefore, the mass transfer, fluid flow and phase transformations were not included in the analysis [24]. The thermal strain was calculated utilising the thermal expansion coefficient of the material and the thermal history of the heating and cooling phases. This was applied to the FE simulation as a predefined field at each corresponding mechanical step. As the melt pool was simulated in the thermal analysis, the melting/solidification of the feedstock and substrate material was accounted for in the mechanical analysis by applying the annealing function, within ABAQUS, to the temperature dependent plastic strain data [25].

\subsection{Validation of the Modelling Strategy}

For this current study, a validation case is presented which implemented the proposed methodology to predict the transient deposition track, thermal history and residual stress field generated by an in-process modification to the laser power. A single track of IN718 was deposited along the centre of an IN718 substrate utilising the following laser power profile. The laser power was fixed at $900 \mathrm{~W}$ for the first 7.5 seconds of the deposition. This value was linearly increased to $1800 \mathrm{~W}$ over the duration of 3 seconds, with the power remaining at $1800 \mathrm{~W}$ for the remainder of the deposition. The total time required to complete the deposition was 18 seconds. The selected power profile was based on the upper and lower 
values of the previously determined process window [26] for the alloy system and the experimental setup adopted for this work. For the entire deposition, the traverse velocity $(V)$ and the powder mass feed rate $(\dot{m})$ were set to a constant value of $400 \mathrm{~mm} / \mathrm{min}$ and 26 $\mathrm{g} / \mathrm{min}$ respectively, based on the work by Abioye et al. [26]. A numerical and experimental case study was conducted to evaluate the validity of the modelling approach.

\subsubsection{Implementation of the Computational Model}

As the thermal field for the track profile model was determined through the use of a transient FE model, a mesh independence study was conducted to determine the optimum element size which represented the heat flux within the domain. The FE domain used was the size of the substrate $(120 \times 70 \times 6 \mathrm{~mm})$ and was meshed with DC3D8 and C3D8R linear brick elements for the thermal and mechanical models respectively. As the melt pool geometry would determine the accuracy of the predicted track profile, this parameter was used as an indicator to determine mesh independence. However, from previous work presented by Walker et al. [16], the temperature field should also be considered to obtain a fully independent mesh. The starting element volume utilised for the mesh study was $2 \times 2 \times 2$ $\mathrm{mm}^{3}$ in the vicinity of the HAZ and was increased through the use of mesh biasing to an element volume of $16 \times 2 \times 2 \mathrm{~mm}^{3}$ at the substrate edge. For each iteration of the study, the element volume was halved and two convergence parameters were calculated based on the current and previous element volume. The coefficient of determination $\left(R^{2}\right)$ for the thermal history profile and a melt pool profile were used as the convergence parameters. The thermal history was extracted from the nodal coordinate $(3,60,0)$ and the melt pool profile was derived from the through thickness temperature data at the substrate mid length. Convergence was achieved when both $R^{2}$ values were greater than or equal to 0.995 as this was consistent with the aforementioned computational model. The study was completed for the maximum and minimum laser powers, i.e. $\mathrm{P}=900 \mathrm{~W}$ and $\mathrm{P}=1800 \mathrm{~W}$, with the results presented in Figure 6. 


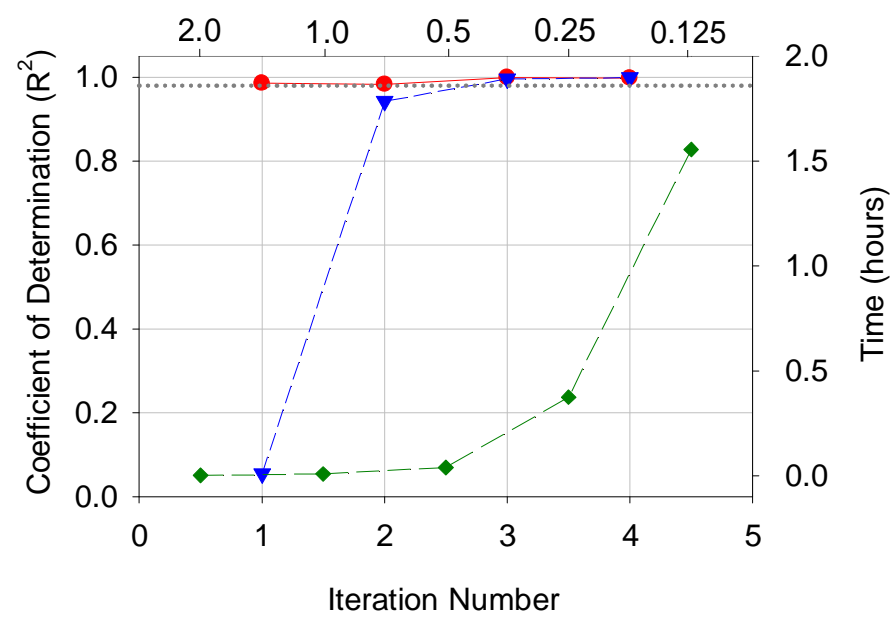

(a)

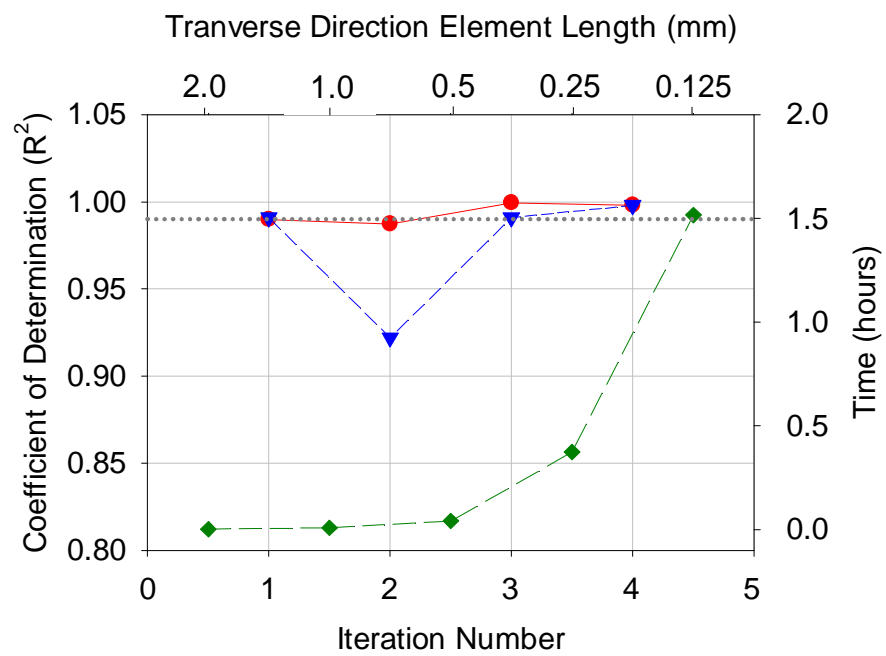

(b)

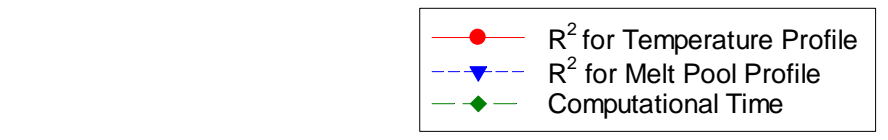

Figure 6 Mesh study results for (a) $P=900 \mathrm{~W}$ and (b) $P=1800 \mathrm{~W}$. Each graph presents the convergence parameters and computational time for each mesh iteration. Convergence criteria is highlighted as a dashed grey line.

It is apparent that minimal variation was present in the accuracy of the thermal history data when the element volume was reduced; therefore, a coarser mesh could be utilised if only thermal data is of interest. Through the use of the second convergence parameter, the accuracy of the melt pool profile is highly dependent upon the resolution of the mesh, as a higher number of iterations were required before convergence was met; therefore, to obtain a more accurate representation of the melt pool geometry, a finer mesh was required. As expected, the computational time increases through reduction of the element volume. The reduction in accuracy observed at iteration 2, for $\mathrm{P}=1800 \mathrm{~W}$ (Figure 6b), arose from increasing the number of nodal points used to represent the through thickness thermal field. This caused the shape of the fusion zone to change from the previous iteration, resulting in a larger melt pool area. For both sets of process parameters, iteration 4 satisfies the 
convergence limits, therefore an element volume of $0.25 \times 0.25 \times 0.25 \mathrm{~mm}^{3}$ was utilised in the vicinity of the heat source, i.e. $-5 \leq \mathrm{x} \leq 5 \mathrm{~mm}$. Outside of the heat source region, i.e. $\mathrm{x} \leq-5$ $\mathrm{mm}$ and $\mathrm{x} \geq 5 \mathrm{~mm}$, an element volume of $1 \times 0.25 \times 0.25 \mathrm{~mm}^{3}$ was utilised. The total number of elements and nodes in the mesh were 622,800 and 656,850 respectively. An example section of the mesh used for the track profile model is presented in Figure 7.

For the thermomechanical model a second mesh was required to include the predicted track geometry. As a variation in the track profile was present, a reduction in element size was required in the transient region. From the mesh sensitivity study (Figure 6), the maximum element volume within the vicinity of the heat source, which allowed for a converged solution, was $0.25 \times 0.25 \times 0.25 \mathrm{~mm}^{3}$. This element volume was utilised for the high-power region, i.e. $\mathrm{P}=1800 \mathrm{~W}$, with the element volume reducing to $0.125 \times 0.125 \times 0.125$ $\mathrm{mm}^{3}$ for the low power region, i.e. $\mathrm{P}=900 \mathrm{~W}$. From Figure 6 a it can be observed that the use of a $0.125 \times 0.125 \times 0.125 \mathrm{~mm}^{3}$ element volume provides a negligible difference in the solution accuracy; therefore, mesh independence was still achieved. Square elements with a constant volume were used throughout the domain to allow the predicted stresses to be volumetrically averaged to compare against the experimental ND data. As the thermal solution was independent of the mesh, an accurate prediction of the transient temperature field was achieved, allowing the residual stress fields to be predicted with accuracy [27]. A total of 717,818 nodes and 672,000 elements were present in the domain. Sections of the mesh from each track region are presented in Figure 8.

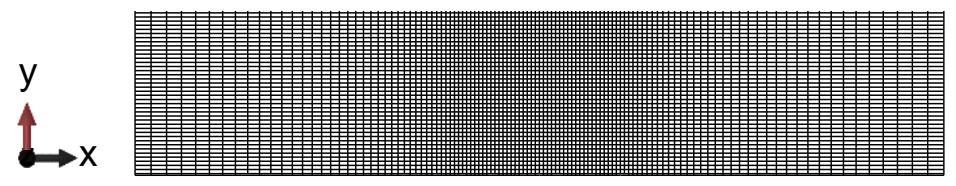

(a)

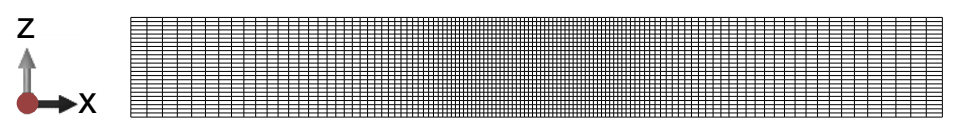

(b)

Figure 7 Final mesh design utilised for the numerical track prediction mode (a) section of the mesh used in the traverse direction; (b) through thickness mesh design. For both images, the laser scanning direction is denoted by the red arrow (y-direction). 


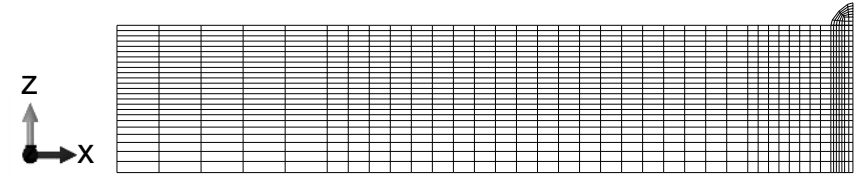

(a)

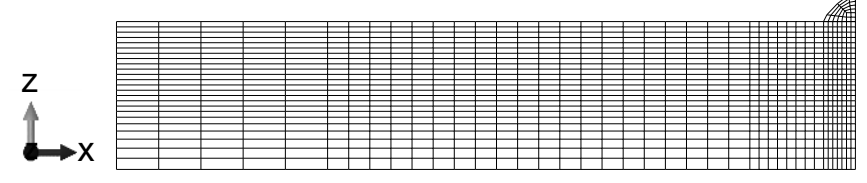

(b)

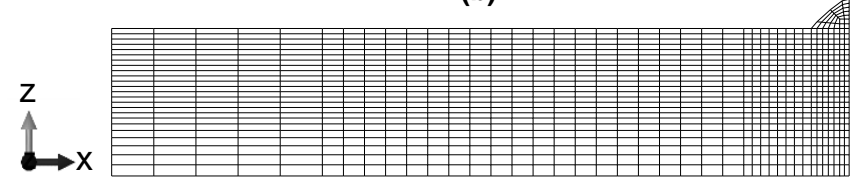

(c)

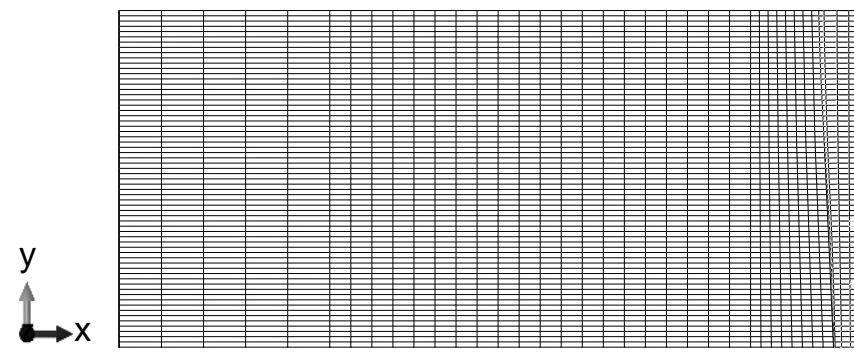

(d)

Figure $8 \mathrm{FE}$ mesh utilised for the thermomechanical model showing the through thickness design at (a) $y=30 \mathrm{~mm}$; (b) $y=60 \mathrm{~mm}$; (c) $y=90 \mathrm{~mm}$; (d) top view of the mesh design in the transient region

Due to the variation in the laser power, the circular disc heat source model was modified for both the track profile model and the thermomechanical model. The FE heat source model therefore had the following form:

$$
\begin{gathered}
Q(x, y, z, t)=\frac{\alpha P(t)-P_{\text {loss }}}{\pi r_{0}^{2}} \exp \left[-\frac{\left(x^{2}+(y-V(t) t)^{2}\right)^{2}}{r_{0}^{2}}\right] \\
\text { for } t \leq t_{s s(1)} \rightarrow P_{\text {loss }}=P_{\text {loss (1) }}^{s s} \\
\text { for } t \geq t_{\text {ss (2) }} \rightarrow P_{\text {loss }}=P_{\text {loss (2) }}^{s s} \\
\text { for } t_{s s(1)}<\mathrm{t}<t_{\text {ss (2) }} \rightarrow P_{\text {loss }}=P_{\text {loss }}(t)
\end{gathered}
$$

where $t_{s s(1)}$ and $t_{s s(2)}$ are the total times for which the deposition was in a steady-state condition, $P(t)$ is the laser power, which was expressed as a function of time when analysing the transient portion of the deposition and $P_{\text {loss }}$ is the total power loss within the system, determined from the track prediction model. The Gaussian radius was assumed to equal half of the measured spot diameter of the laser, which took a value of $1.3 \mathrm{~mm}$ in this work. From 
geometrical measurements of the nozzle (Table 1) and assuming that the material properties of the powder are equal to the substrate properties, the Gaussian feed radius was calculated as $2.0 \mathrm{~mm}$ for this study. Through implementing Eq.(8), the power loss for both the steady-state track regions $\left(P_{\text {loss (1) }}^{S S}\right.$ and $\left.P_{\text {loss (2) }}^{S S}\right)$ was constant due to the melt pool having a fixed width and length in this region. Therefore, the power loss in these regions were represented within the FE heat source model as a single value at the corresponding time steps; however, in the transient region, due to the changes in the melt pool dimensions at each time increment, the power loss function $\left(P_{\text {loss }}(t)\right)$ was defined within the user subroutine DFLUX.

Table 1: Geometrical parameters for the coaxial nozzle used for this study.

\begin{tabular}{lc}
\hline \multicolumn{1}{c}{ Parameter } & Dimension \\
\hline Nozzle stand-off distance $\left(Z_{s}\right)$ & $12 \mathrm{~mm}$ \\
Nozzle inclination angle $(\zeta)$ & $90^{\circ}$ \\
Annulus distance $\left(r_{0}-r_{i}\right)$ & $0.25 \mathrm{~mm}$ \\
Initial powder stream angle $(\theta)$ & $60^{\circ}$ \\
Powder stream divergence angle $(\phi)[20]$ & $8^{\circ}$ \\
\hline
\end{tabular}

As different melt pool sizes were present throughout the analysis, the number of elements reactivated to simulate the deposition of material, per time step, was not uniform. This was required to ensure that a stable energy balance within the melt pool itself was achieved. For the region where $\mathrm{P}=900 \mathrm{~W}$, two element lengths per time step were reactivated, in all other regions a total of four element lengths were reactivated.

For all of the numerical FE models, temperature dependent material properties were utilised, as presented in Figure 9. Poisson's ratio, was assumed to be independent of temperature, with a value of 0.33 [28]. The latent heat of melting was included for the respective solidus $\left(1260^{\circ} \mathrm{C}\right)$ and liquidus $\left(1336^{\circ} \mathrm{C}\right)$ temperatures. The thermal properties used to implement the analytical expressions (eq.5 and eq.14) for the track profile model are presented in Table 2. The density and specific heat capacity were assumed to be temperature independent defined at $630^{\circ} \mathrm{C}$.

Table 2: Thermal properties for IN718 used to determine the track profile.

\begin{tabular}{ll}
\hline Density $\left(\mathrm{kg} / \mathrm{m}^{3}\right)[28]$ & 7993 \\
Specific Heat Capacity $(\mathrm{J} / \mathrm{KgK})[28]$ & 536 \\
Latent Heat of Melting $(\mathrm{J} / \mathrm{Kg})[29]$ & 210 \\
Latent Heat of Vaporisation $(\mathrm{kJ} / \mathrm{Kg})[30]$ & 6400 \\
Solidus Temperature $\left({ }^{\circ} \mathrm{C}\right)[29]$ & 1260 \\
Liquidus Temperature $\left({ }^{\circ} \mathrm{C}\right)[29]$ & 1336 \\
Laser Absorptivity [31] & 0.3 \\
\hline
\end{tabular}


The material properties were assumed to be equal for the substrate and track geometry. Although there will be metallurgical differences in the grain size and the microstructure between the HAZ, track region and far field in the substrate, this assumption is valid from the work presented by Deshpande et al. [32]. Alteration of the stress fields due to phase changes were neglected from the mechanical model, as no allotropic phase transformations occur for IN718 at elevated temperatures [33]. As no solid state transformations are present between the ambient temperature and the melting temperature of IN718 [34], the transformation strains are not included. This was supported by Denlinger and Michaleris [35], where the effect of phase transformations had no effect on the final residual stress field for IN718. Therefore, the total strain $\left(\varepsilon_{T}\right)$ consisted of three strain components for the FE solution:

$$
\varepsilon_{T}=\varepsilon_{e}+\varepsilon_{p}+\varepsilon_{t h}
$$

where $\varepsilon_{e}$ is the elastic strain component modelled using an isotropic Hooke's law material model, $\varepsilon_{p}$ is the plastic strain which was determined using the von Mises yield criterion and $\varepsilon_{t h}$ is the thermal strain. The plastic strain was rate independent, with an isotropic hardening coefficient defined as $0.01 E$ [28]. During a single track deposition, no thermal cycling is present because no reheating of previously deposited tracks is present, therefore, based on the work by Muransky et al. [36], the isotropic hardening law is considered to be valid. It should be noted that the stress relaxation effect, which could occur from applying thermal loads to pre-stressed structures, was not included within the mechanical model. From previous work presented by Salerno et al. [37], stress relaxation was not observed for IN718, when welding of a pre-stressed component was completed. A reduction in residual stress was observed due to mechanical redistribution of the stress and this was captured without inclusion of a mechanism to account for stress relaxation.

The convective heat transfer coefficient and emissivity were set to $25 \mathrm{~W} / \mathrm{m}^{2} \mathrm{~K}$ and 0.8 respectively [32]. The artificial convective boundary condition was defined with a high heat transfer coefficient, taking a value of $800 \mathrm{~W} / \mathrm{m}^{2} \mathrm{~K}$ [38]. As no fixed clamping mechanisms were used in the experimental set up, the mechanical boundary conditions were set to only restrict rigid body motion and to reflect the conditions at the symmetry plane $\left(x=0, R_{y}=\right.$ $0, R_{z}=0$ ). The mechanical analysis was solved without taking into consideration large displacements or the resulting geometric non-linearity. 


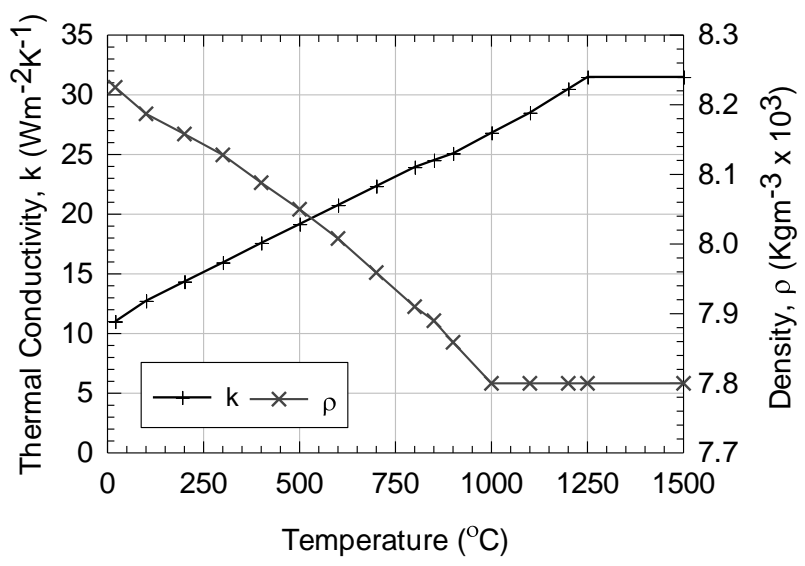

(a)

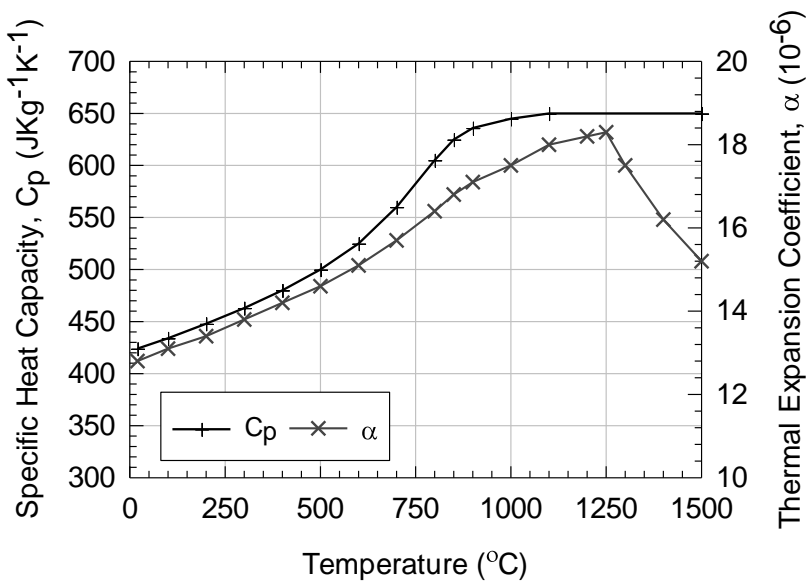

(b)

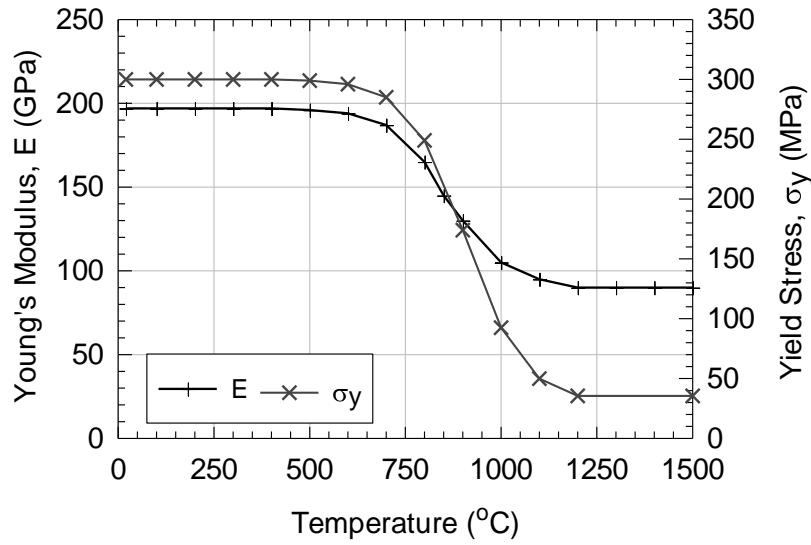

(c)

Figure 9 Temperature dependent thermomechanical properties of IN718 used for all FE models (a) Thermal conductivity, $k$, and density, $\rho$ (b) Specific heat capacity, $C_{p}$, and thermal expansion coefficient, $\alpha$ (c) Young's modulus, $E$, and yield stress, $\sigma_{y}$ [28].

\subsubsection{Experimental Validation}

A single track of IN718, consisting of a varying cross-sectional geometry, was deposited utilising a variation in the laser power profile as outlined previously. Two IN718 plates (120 
x $70 \times 6 \mathrm{~mm}$ ) were used for the substrate material and were supplied sand-blasted in the solution heat treated state by Haynes International (Manchester, UK.). The chemical composition of the material is presented in Table 3. A $2 \mathrm{~kW}$ Ytterbium doped, continuous wave, fibre laser (IPG Photonics) operating at a wavelength of $1070 \mathrm{~nm}$ was used. A Precitec YC 50 cladding head, fitted with a coaxial deposition nozzle, delivered powder to the substrate using argon as the carrier gas. The laser beam was operated in-focus, which produced a $2.6 \mathrm{~mm}$ spot diameter. A Praxair (Model 1264) powder feeder was used to deliver gas atomised MetcoAdd 718C powder to the substrate with a nominal size ranging from $-45 \mu \mathrm{m}$ to $15 \mu \mathrm{m}$ [39]. The argon carrier gas was set to $10 \mathrm{~L} / \mathrm{min}$ and the mass flow rate of powder was calibrated to $26 \mathrm{~g} / \mathrm{min}$.

Table 3: Chemical Composition of IN718 in wt \%.

\begin{tabular}{cccccccccccc}
\hline Ni & Co & Fe & Cr & Co+Ta & Mo & Mn & Si & Ti & Al & C & B \\
\hline 52 & 1 & 19 & 18 & 5 & 3 & 0.35 & 0.35 & 0.9 & 0.5 & 0.05 & 0.004 \\
\hline
\end{tabular}

Two identical samples denoted sample A and sample B were manufactured, with a schematic of the experimental set up shown in Figure 10. The coordinate system remained constant throughout, with the build direction being in the positive $z$ axis and the $x-y$ axis forming a plane perpendicular to the build direction. Sample A was used for in-situ temperature measurements, metallurgical examination and for the ND measurements. To obtain the elastic strain within sample A using ND, a strain-free reference value for the lattice spacing $\left(d^{0}\right)$ in each region of interest was required [40]. Sample B was used to fabricate a comb specimen (detailed below) required to measure the strain-free reference value through ND measurements. To prevent rigid body motion during the fabrication of both samples $A$ and $B$, four equispaced restraining bolts were positioned along the width and the length of the substrate. No other clamping mechanisms were used in the experimental set up. It should be noted that to ensure a track was formed instantaneously upon the laser being activated, the powder feed was switched on before the laser as highlighted in Figure 10. The full track length equated $110 \mathrm{~mm}$ and upon its completion, the sample was cooled in air to room temperature. 


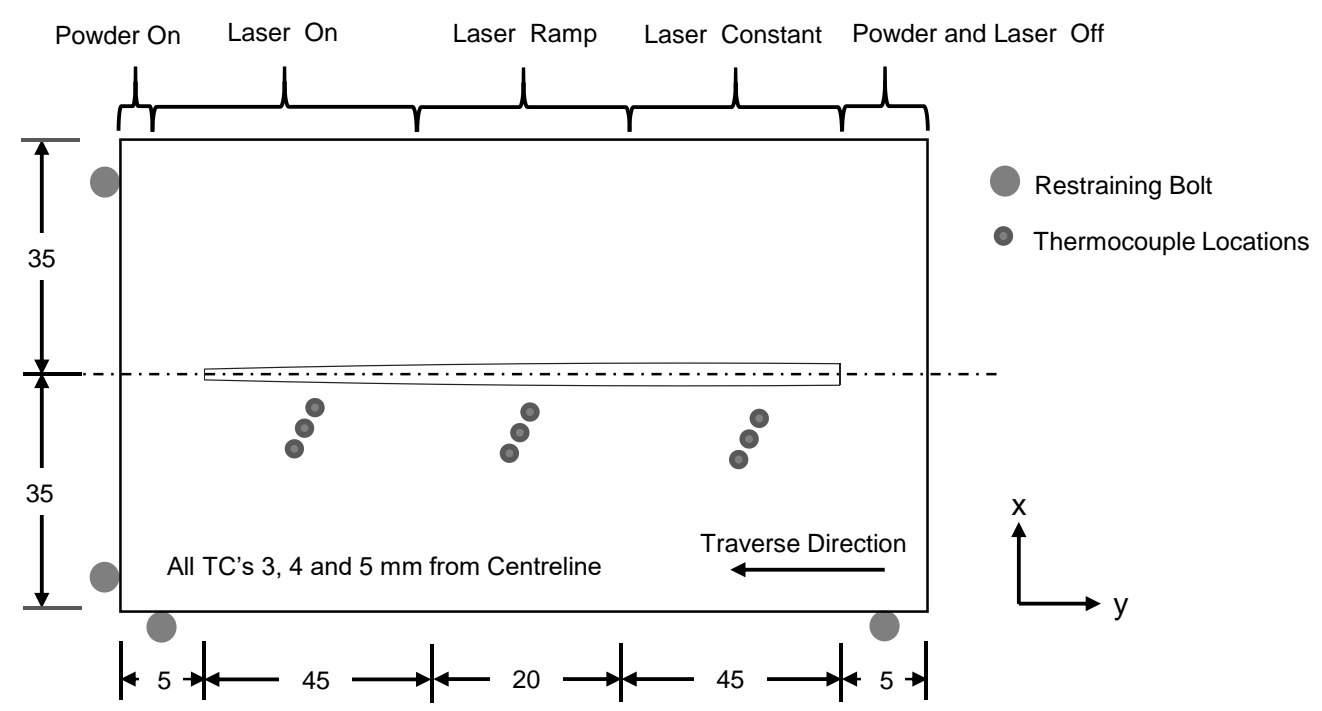

Figure 10 Schematic of the experimental set up showing the locations of the restraining bolts, thermocouples used to collect thermal histories and the order in which the laser and powder feed were activated/deactivated.

A total of nine in-situ temperature measurements were recorded in the region of the Heat Affected Zone (HAZ) using K-type thermocouples. Each thermocouple was spot welded to the upper surface of the substrate, using an open junction arrangement, at a distance of $y=30$ $\mathrm{mm}, 60 \mathrm{~mm}$ and $90 \mathrm{~mm}$. The positioning of the thermocouples relative to the substrate are shown in Figure 10. Data was captured using a National Instrument SCXI-1000 chassis in conjunction with a SCXI-1102B voltage input module.

To verify the predicted melt pool geometry, metallographic analysis was conducted on sample A. Specimens were removed from each of the three regions of the track by waterjet cutting, as shown in Figure 11. Each of the three specimens were mounted in conductive resin using a $30 \mathrm{~mm}$ press. Each specimen was ground using $\mathrm{SiC}$ abrasive paper to a 1200 grit size, then polished using a 6 and $1 \mu \mathrm{m}$ diamond wheel. To reveal the fusion zone of the material, the specimen was etched using Marble's Reagent (10g CuSO ${ }_{4} 50 \mathrm{ml} \mathrm{HCL}$ and $50 \mathrm{ml}$ $\mathrm{H}_{2} \mathrm{O}$ ). The etchant was swabbed onto the exposed surface of the specimen and removed after no longer than 10 seconds. Optical microscopy was used to examine each specimen. To determine the experimental track geometry, images of the top surface of the substrate were measured using focus variation microscopy. An Alicona InfinteFocus fitted with a 5x optical lens was used. The entire top surface was scanned, with cross sectional profiles being extracted from the image at $5 \mathrm{~mm}$ intervals. 


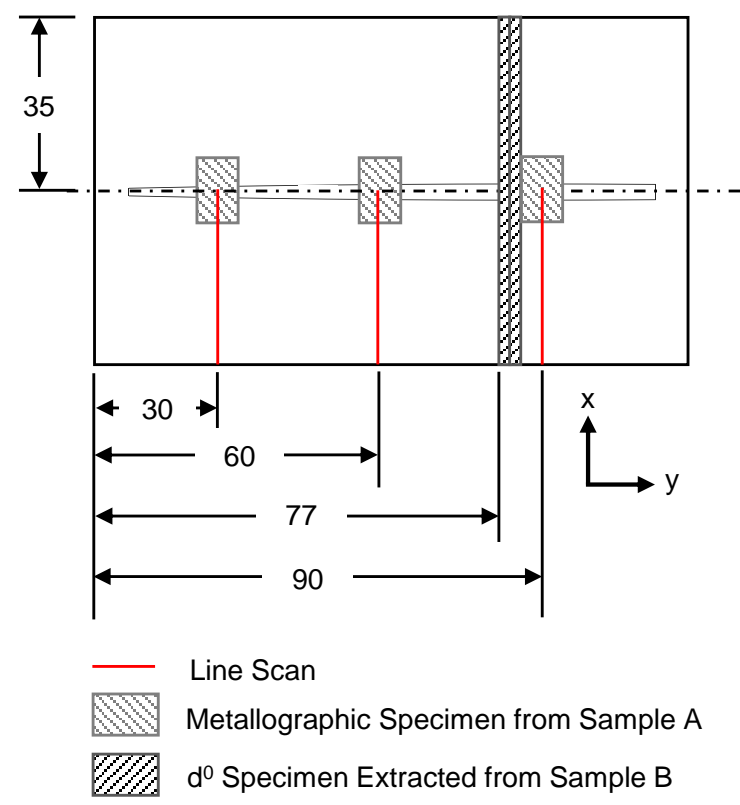

Figure 11 Locations of the three specimens extracted from sample A used for metallographic examination. Horizontal line scan locations used for the ND measurements shown in red.

ND measurements were completed at the ENGIN-X facility using the ISIS neutron source located at the Rutherford Appleton Laboratory, UK [41]. Two strain components were collected simultaneously using two directly opposing detector banks at $2 \theta= \pm 90^{\circ}$. Three line-scans were taken at three locations along the length of the sample (Figure 11). All measurements were conducted at $2 \mathrm{~mm}$ below the surface of the substrate using a gauge volume of $2 \times 2 \times 2 \mathrm{~mm}^{3}$. Through rotation of the sample, a full triaxial stress analysis was conducted. To quantify the high magnitude residual stresses expected in the region of the track, an increased spatial resolution (smaller spacing between ND measurement positions) was used. To obtain the strain-free reference measurements, comb shaped specimens [40], as shown in Figure 12, were manufactured using Electro Discharge Machining (EDM). The same spatial resolution used to acquire the strain-free lattice spacing was also used for the measurements in sample A, therefore two $d^{0}$ specimens (4 $\mathrm{mm}$ Thick) were required to ensure that the gauge volume could be situated within each tooth of the comb. The specimens were removed from sample $B$ at $y=77 \mathrm{~mm}$ and $\mathrm{y}=81 \mathrm{~mm}$ (Figure 11). It should be noted that the beam time allocation did not allow for a strain-free reference sample to be measured at each line scan location. 


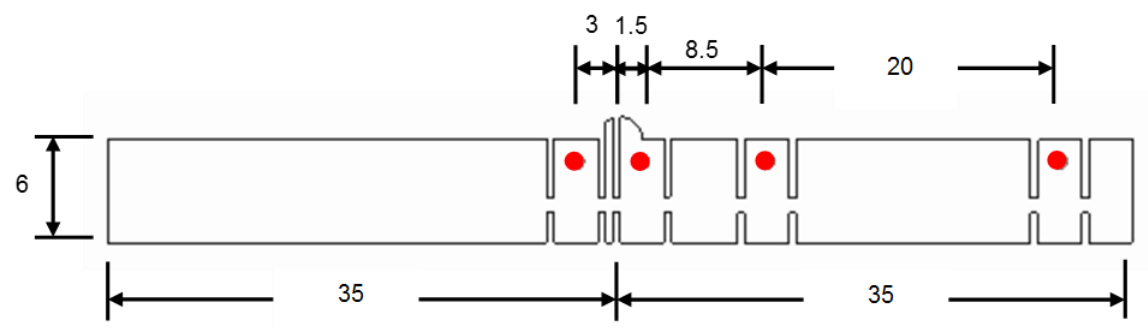

(a)

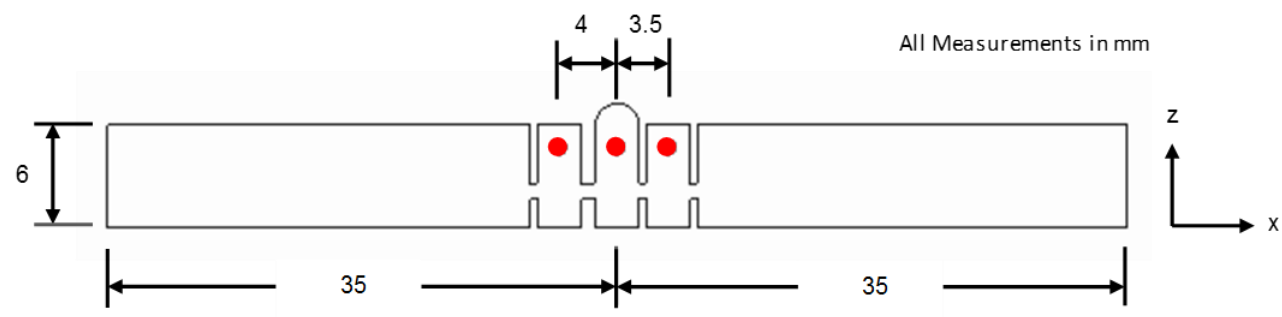

(b)

Figure 12 Details of the strain free reference ( $d^{0}$ ) specimen's after EDM (a) d ${ }^{0}$ sample one (b) d ${ }^{0}$ sample two. Red circles mark the target location of the beam for the ND measurements.

The Rietveld refinement method was used to determine the lattice spacing implementing the General Structure Analysis System (GSAS) to process and analyse the ND data. The elastic lattice strain was calculated as follows:

$$
\varepsilon=\frac{d-d^{0}}{d^{0}}
$$

where $d^{0}$ is the strain free lattice spacing and $d$ is the lattice spacing of interest. The macroscopic stress components in the $i$ direction can be obtained from Hooke's law:

$$
\sigma_{i j}=\frac{E}{1+v}\left[\varepsilon_{i j}+\frac{v}{1-2 v}\left(\varepsilon_{11}+\varepsilon_{22}+\varepsilon_{33}\right)\right]
$$

where the numerical subscripts denote the direction in the sample, $E$ is the Young's modulus and $v$ is Poisson's ratio, with values of $209 \mathrm{GPa}$ and 0.29 respectively for IN718 [42]. Through using GSAS, the uncertainty from fitting the nominal diffraction peaks to the measured diffraction spectra were calculated for both the strain-free lattice parameter $\left(d^{0}\right)$ and the lattice spacing of interest $(d)$. Through propagation of uncertainty, a combined error analysis, using Eqs.(1) and (2), can be conducted to determine the uncertainty in the experimental stress for all three macroscopic stress components. 


\section{Results}

\subsubsection{Numerical Track Prediction}

A contour map of the deposited track, created from 25 profiles extracted from the focus variation microscopy image, is presented in Figure 13. An average profile was determined for the two steady-state regions, these are compared to the predicted profiles in Figure 14. For the transient region, an average profile was not determined due to the evolving track geometry. The comparisons of the two data sets for this region are shown in Figure 15. The error and coefficient of determination $\left(R^{2}\right)$ between both data sets is presented in Table 4 . From analysing both sets of data, the track width and height increase during the transient phase, reaching a steady-state profile at the end of the transient region. However, the experimental track width appears to reach a constant value before the end of the transient region. The predicted shape of the track profile in all three regions agrees well with the experimental data, as the majority of the $R^{2}$ values are greater than $90 \%$, showing confidence in the predicted values. It should be noted that for $y=60 \mathrm{~mm}$ and $\mathrm{y}=65 \mathrm{~mm}$, the experimental bead geometry was asymmetric, therefore the error between the track height and width maybe due to the model not accounting for this phenomena. The numerical model predicts the track profile with a greater accuracy at the low laser power. During the transient phase of the process, the height is captured well by the model; however, the predicted track width is overestimated when compared to the experimental data. The maximum error between the predicted and experimental data arises at the final transient profile and for the profile used in the second steady-state region. In these regions, the peak height of the profile has been predicted with accuracy, therefore the error must arise from the derivation of the melt pool width.

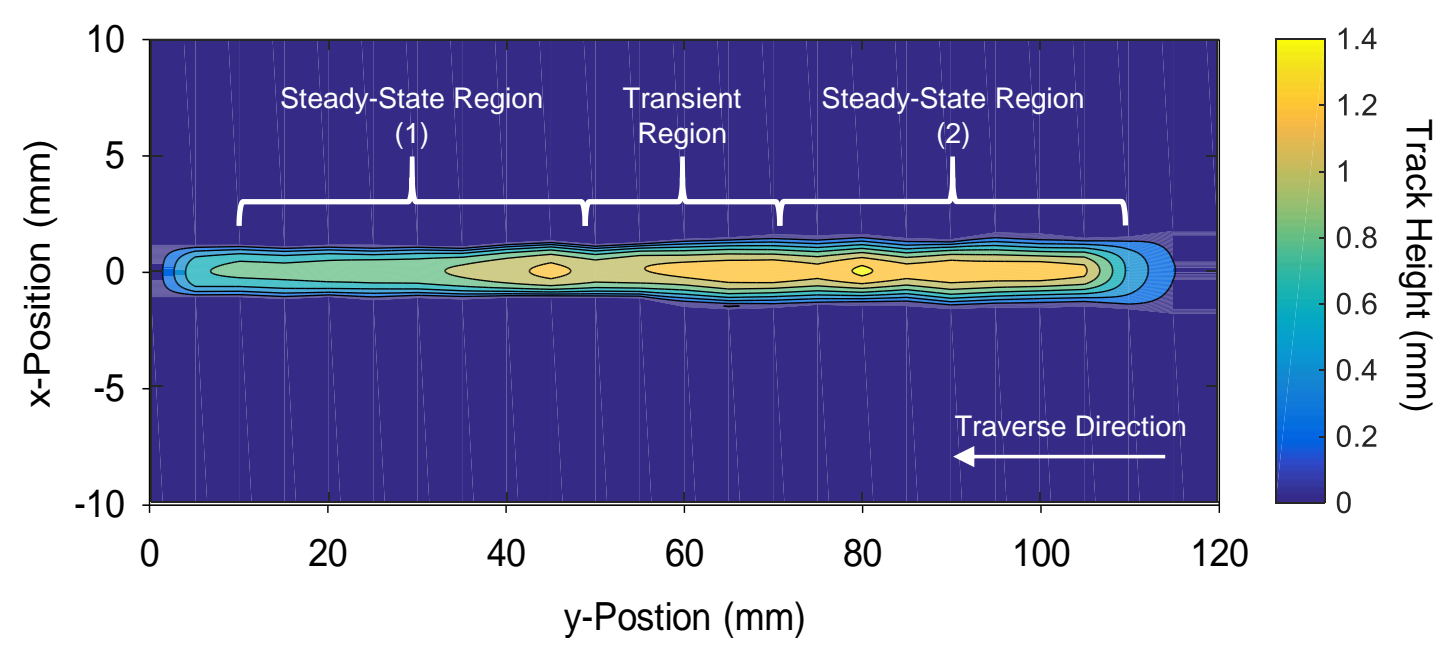

Figure 13 Contour plot showing the variation in track height derived from the surface variation microscopy data with the three distinct track regions have been highlighted. 


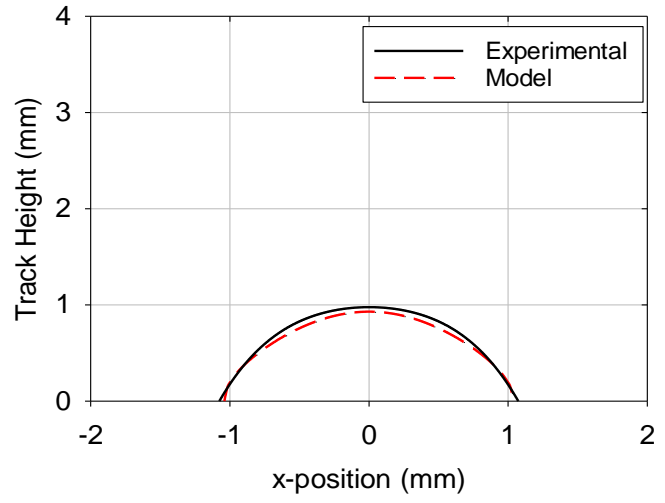

(a)

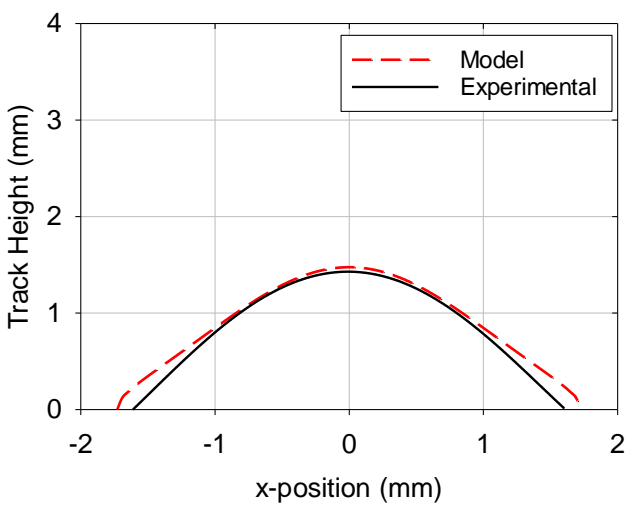

(b)

Figure 14 Comparison of the experimental and predicted track geometry for two laser powers (a) $P=900 \mathrm{~W}$ and (b) $\mathrm{P}=1800 \mathrm{~W}$.
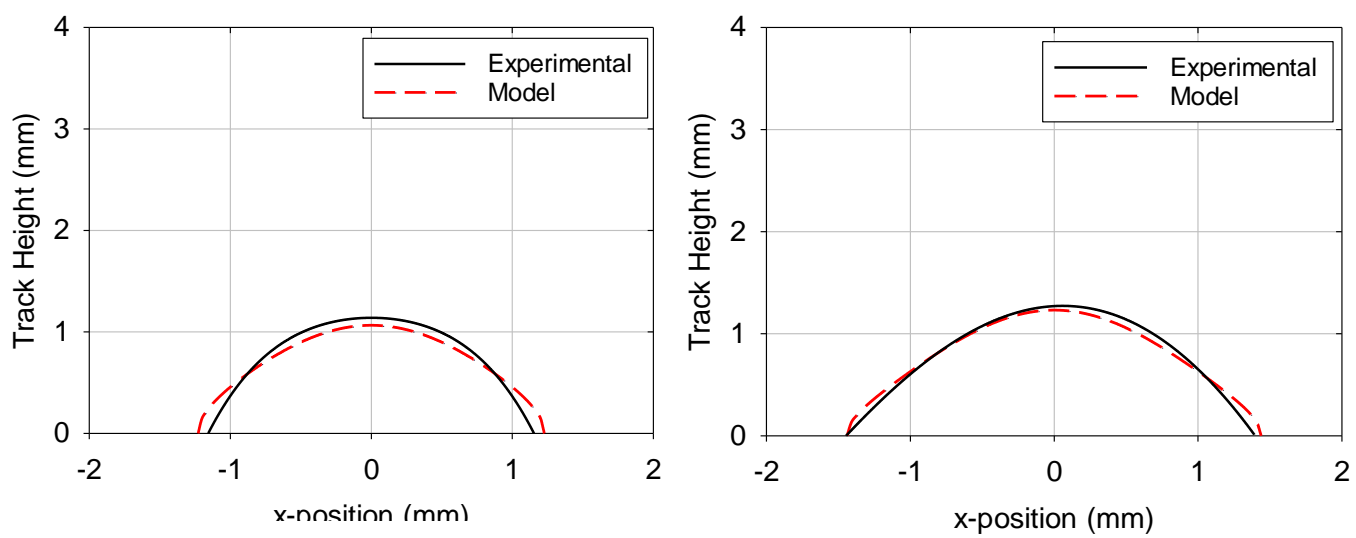

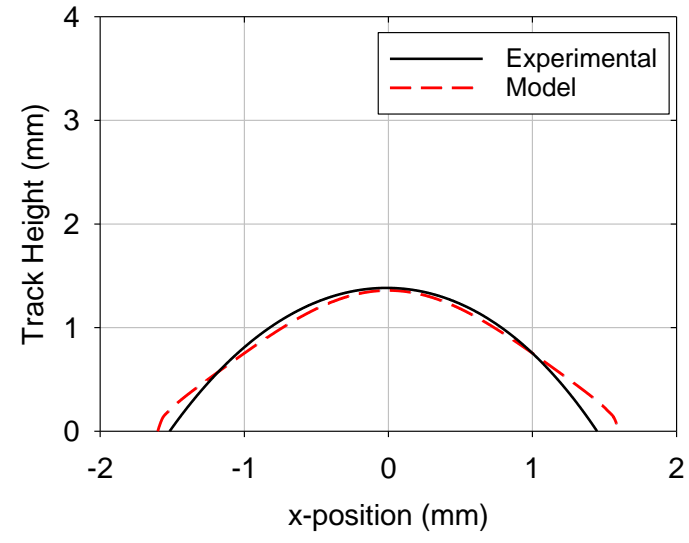

(c) (b)

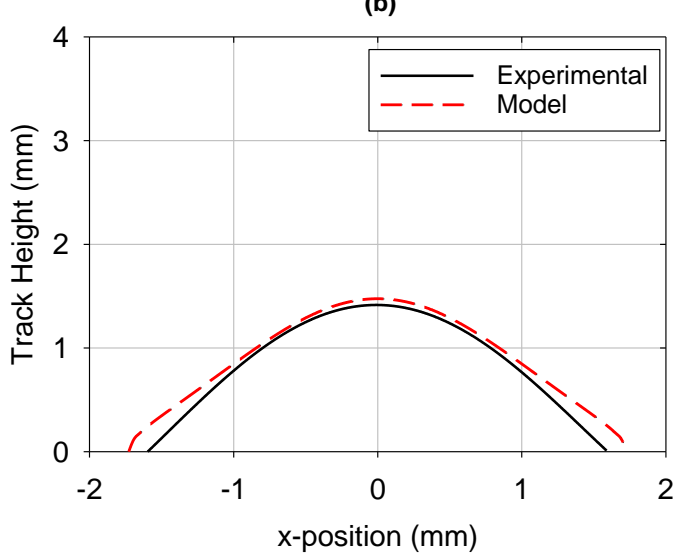

(d)

Figure 15 Comparison of the experimental and predicted track geometry at four locations within the transient region. (a) $y=55 \mathrm{~mm}$ (b) $y=60 \mathrm{~mm}$ (c) $y=65 \mathrm{~mm}$ (d) $y=70 \mathrm{~mm}$. 
Table 4 Error and $\boldsymbol{R}^{2}$ Values for Experimental and Predicted Track Profiles

\begin{tabular}{cccc}
\hline Distance $(\mathrm{mm})$ & Error $W_{\max }(\%)$ & Error $h_{\max }(\%)$ & $\begin{array}{c}\text { Coefficient of } \\
\text { Determination }\end{array}$ \\
\hline $5 \leq \mathrm{y} \leq 50$ & 3.79 & 4.81 & 0.962 \\
55 & 5.38 & 6.50 & 0.934 \\
60 & 7.08 & 4.38 & 0.921 \\
65 & 3.47 & 1.77 & 0.937 \\
70 & 7.62 & 4.25 & 0.992 \\
$75 \leq \mathrm{y} \leq 115$ & 6.73 & 3.25 & 0.994 \\
\hline
\end{tabular}

The power loss history and effective power utilised in the transient portion of the numerical FE model is presented in

Figure 16. For the steady-state regions, i.e $\mathrm{P}=900 \mathrm{~W}$ and $\mathrm{P}=1800 \mathrm{~W}$, the power loss $\left(P_{\text {loss }}^{\text {ss }}\right)$ was calculated as $71.1 \mathrm{~W}$ and $165.7 \mathrm{~W}$ respectively. The power loss over the duration of the deposition follows a linear trend, with a greater reduction in the effective power observed for an increase in the laser power. This is due to the melt pool geometry enlarging at each time step; therefore leading to an increase in the mass of powder which is being assimilated by the melt pool, resulting in a higher energy requirement to sustain mass addition. This therefore reduces the power available at the substrate, which is represented by the effective power in the model.

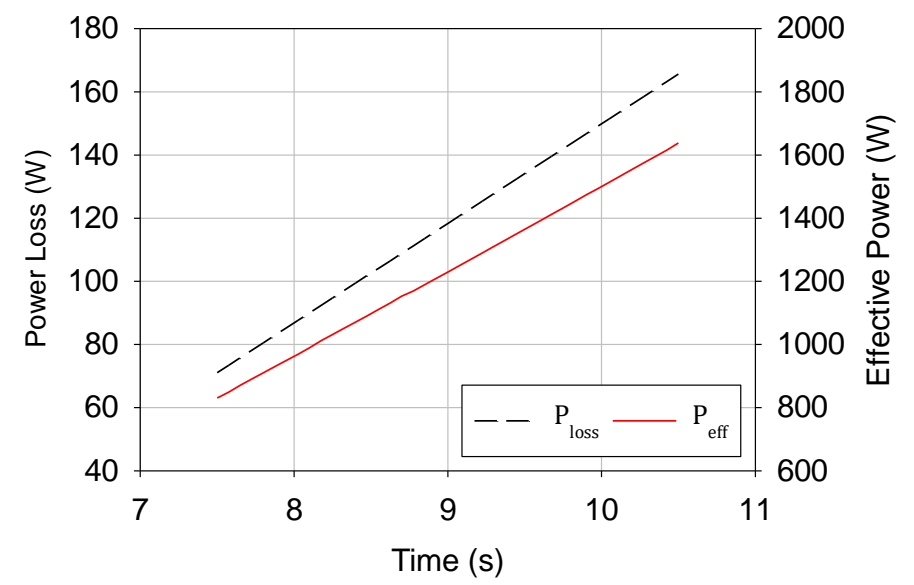

Figure 16 Predicted power loss and effective power utilised during the transient portion of the model.

The catchment efficiency used for the transient portion of the model is presented in Figure 17. For the steady-state regions, the calculated value for the catchment efficiency was 0.38 and 0.48 for $\mathrm{P}=900 \mathrm{~W}$ and $1800 \mathrm{~W}$ respectively. The overall trend for the transient catchment efficiency was approximately linear. At higher laser powers, the melt pool geometry increases in size due to the increase in heat energy available at the substrate; therefore, the 
total amount of powder which can be assimilated will rise as the melt pool covers a greater portion of the powder flux distribution at the substrate.

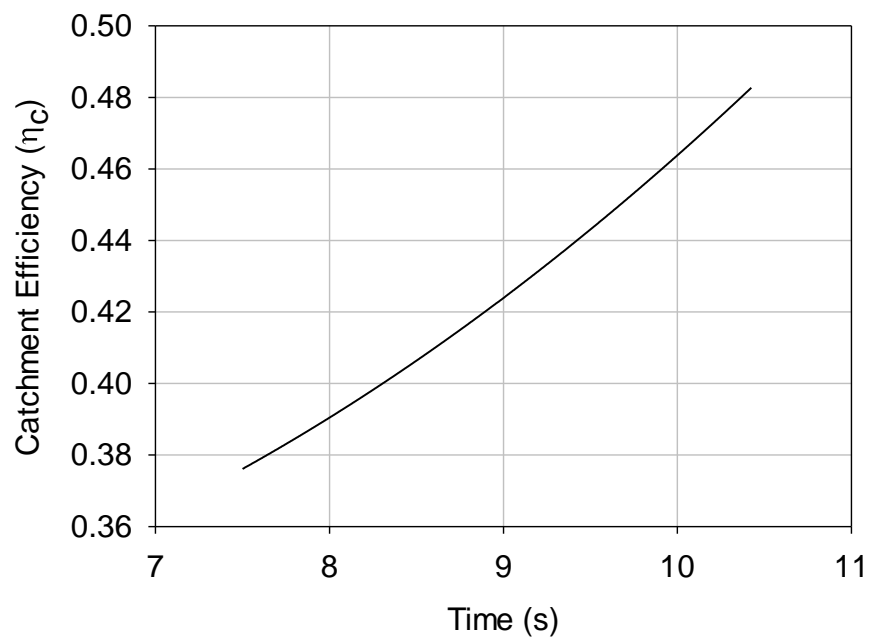

Figure 17 Predcited catchment efficiency used during the transient portion of the deposition.

\subsubsection{Thermal Analysis}

In-situ temperature measurements were compared with the thermal histories from the FE model as shown in Figure 18. For all three locations, the overall trend has been replicated well. The peak temperatures for all thermocouple locations have been overestimated by the FE model, with a maximum error of $6.2 \%$ in the transient region. The predicted thermal gradient prior to the heat source pass, for all three regions, has a sharper gradient than expected when compared to the experimental data. For the thermocouple measurements at $\mathrm{y}=60 \mathrm{~mm}$, there are fluctuations in the temperature collected by the far most thermocouple. This could be due to noise or as a result of the junction not being in contact at $\mathrm{x}=5 \mathrm{~mm}$, potentially leading to the difference observed in the peak temperature value at this thermocouple location. In the cooling phase of the model, the artificial boundary condition, in conjunction with the convective and radiative losses, provides a good estimate of the cooling rate after the heat source passes the measurement location.

A comparison of the predicted weld pool and the experimental micrograph are compared for each of the three track regions in Figure 19. The isotherms presented were taken at the widest point at each location investigated. The grey region represents the fusion zone with contour restricted to the solidus temperature $\left(1260^{\circ} \mathrm{C}\right)$. Overall a fair agreement between the predicted and experimental weld pool is apparent at all three locations. However, the greatest deviation between the two data sets is for the weld pool depth at $\mathrm{y}=35 \mathrm{~mm}$ and $\mathrm{y}=65$ $\mathrm{mm}$. Overall, the shape of the fusion zone has been captured with accuracy, with the variation in penetration depth and melt pool width being captured at all three locations. It can be observed, that through the use of a laser heat source, the heating effects to the substrate are minimal due to the reflectivity of the material. For the predicted data set in 
both the transient and high laser power region, the melt pool geometry no longer encompasses the track material. However, it can be observed in Figure 19b and Figure 19c that this happens in reality due to a layer of powder adhering to the track surface. The layer of powder varies from a thin layer at the low laser power and increases in thickness throughout the deposition. 


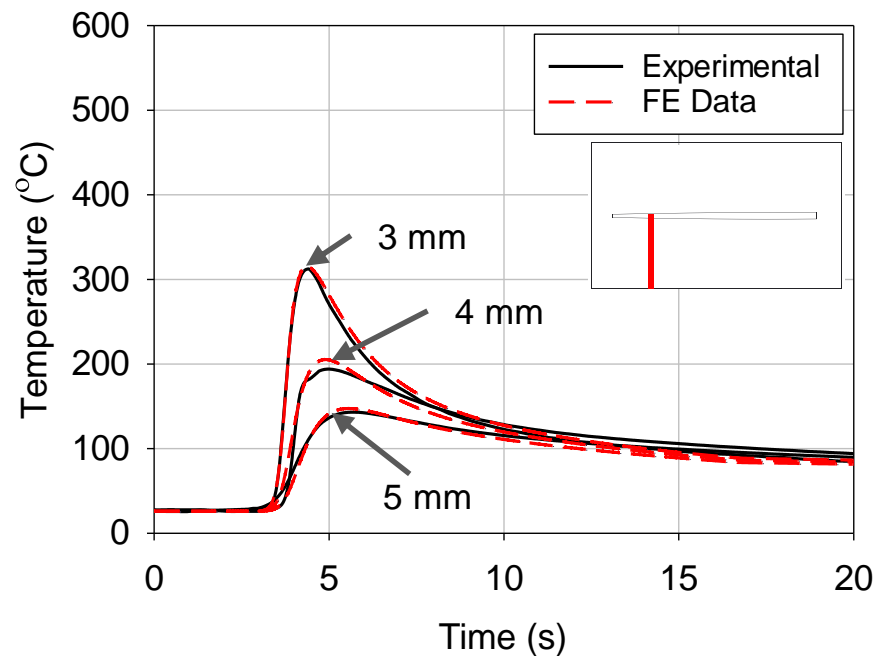

(a)

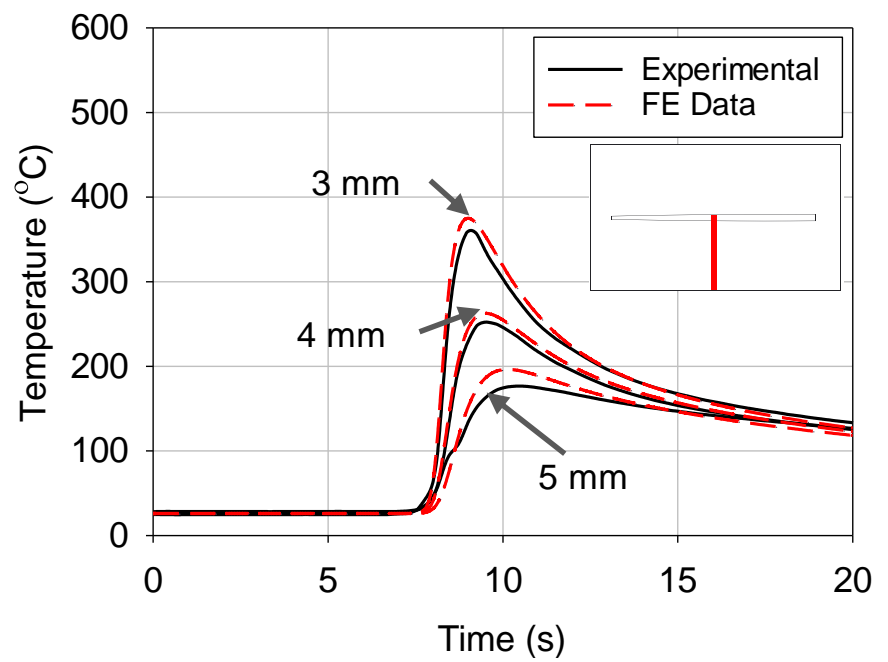

(b)

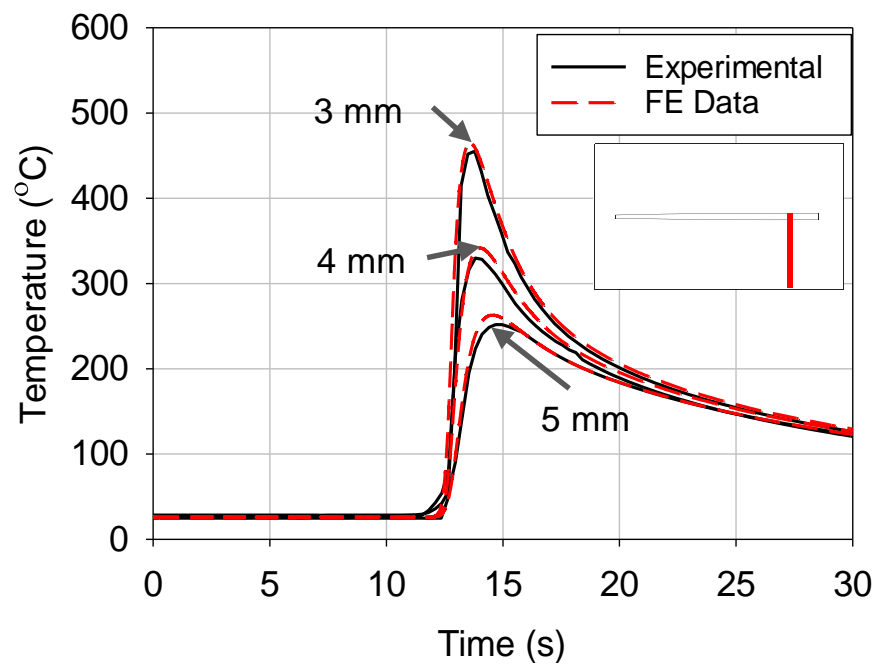

(c)

Figure 18 Comparison of experimental and numerically predicted thermal histories at three locations along the substrate at (a) $y=30 \mathrm{~mm}$; (b) $y=60 \mathrm{~mm}$ and (c) $y=90 \mathrm{~mm}$ for three thermocouple's positioned at $3 \mathrm{~mm}, 4 \mathrm{~mm}$ and $5 \mathrm{~mm}$ from the track centreline. 


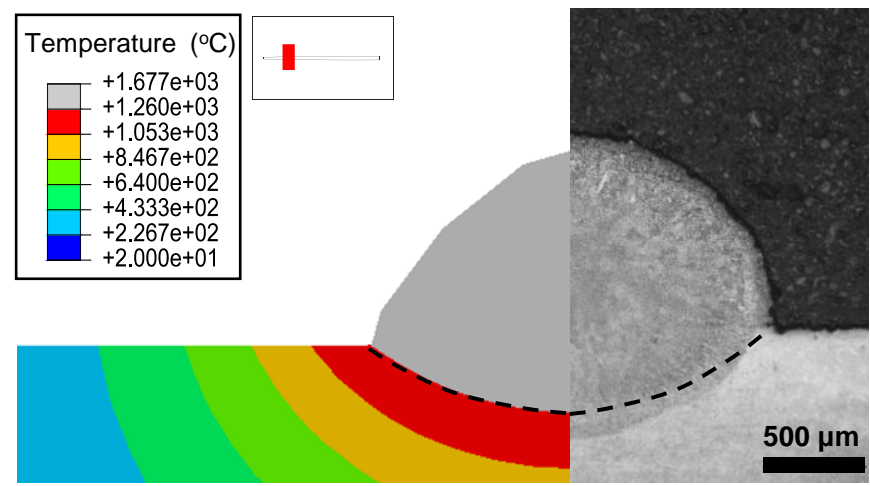

(a)

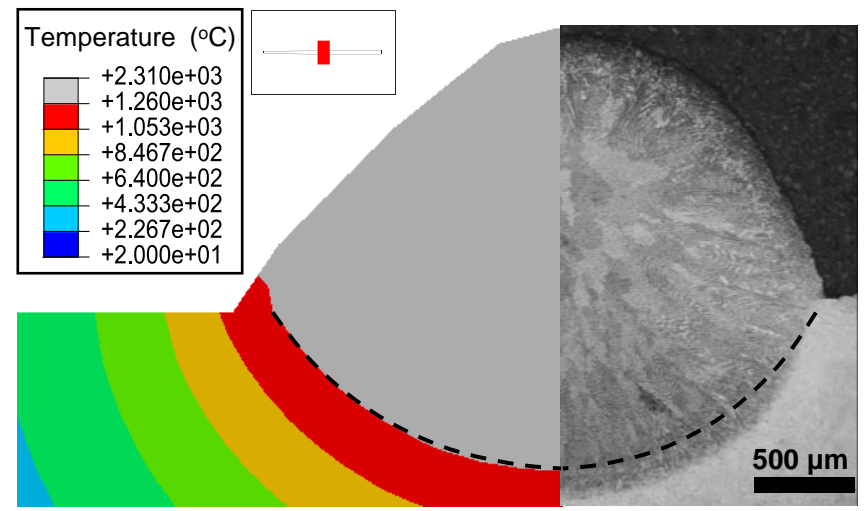

(b)

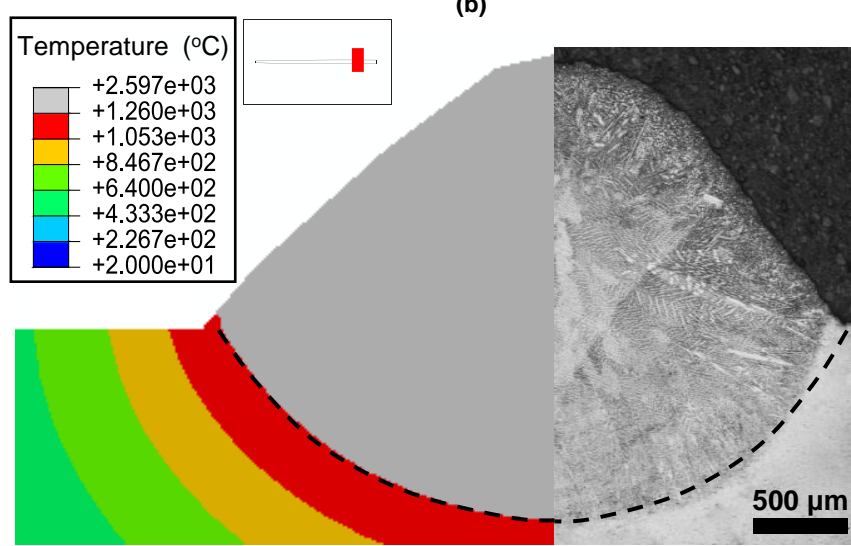

(c)

Figure 19 Comparison of the numerically predicted fusion zone and the metallographic specimen at three locations (a) $y=35 \mathrm{~mm}$; (b) $y=65 \mathrm{~mm}$ and (c) $y=95 \mathrm{~mm}$.

\subsubsection{Mechanical Analysis}

The evolution of stress at a position in the melt pool for each track region is presented in Figure 20. Prior to the heat source pass, the stress state is not in equilibrium, due to the evolution of a compressive and tensile stress for the longitudinal and transverse components respectively. The normal stress component is zero until the peak temperature is reached. As the temperature approaches the melting temperature $\left(1260^{\circ} \mathrm{C}\right)$ of the material, all three stress components, at all three locations, become compressive. The magnitude of the compressive stress upon reaching the peak temperature is constant at all three locations, therefore, the stress evolution directly below the track is independent of the size of the melt 
pool. Shortly after the peak temperature is achieved, all three components of stress enter a tensile state, at the same rate, for all three locations. Upon cooling the component to room temperature, the time required to reach the final residual stress state reduces with an increase in laser power. In the region where $\mathrm{P}=1800 \mathrm{~W}$, the transverse stress reaches a peak value and does not decay to a lower value upon cooling. This arises due to the increased cooling rate $(-d T / d t)$ observed in the high laser power region $\left(2150^{\circ} \mathrm{C} / \mathrm{s}\right)$ compared to the low laser power region $\left(1560^{\circ} \mathrm{C} / \mathrm{s}\right)$; therefore maintaining the current stress state within the component. At room temperature, the longitudinal and transverse stresses are tensile, with varying magnitudes at each location. The variation in stress magnitude occurs due to the evolution of the melt pool geometry at each location, this dictates the amount of expansion and contraction of material occurring in front and behind the heat source. The normal component returns to approximately zero for the low laser power; however, an increase in magnitude is observed with an increase in laser power.

To validate the mechanical analysis, a comparison between residual stress predictions and Neutron Diffraction (ND) measurements was conducted for all three horizontal line scans. Figure 20 shows the two data sets for the longitudinal, transverse and normal stress directions. A good agreement between the experimental and predicted data sets is apparent for all three stress components. The majority of predicted stress values fall within the experimental error showing a high degree of confidence in the results. Due to the variation in the laser power, a higher magnitude longitudinal stress is present in the high laser power region compared to the low power region; therefore, following the expected trend. This component of stress, for all three locations, follows the typical trend of a traditional single weld. The transverse stress component in the track vicinity is of equal magnitude at all three locations, exhibiting a tensile stress state. At the location of $\mathrm{y}=30 \mathrm{~mm}$ and $60 \mathrm{~mm}$, a sharp stress gradient is present in the vicinity of the track for the transverse stress component. In this location, a compressive stress is observed; however, this is not present for $\mathrm{y}=90 \mathrm{~mm}$ as the transverse stress profile remains tensile for the entire width. The normal stress follows the same trend as the transverse component at each horizontal location. The largest error occurs below the track at $\mathrm{y}=30 \mathrm{~mm}$, for the transverse stress, as a greater magnitude was measured than predicted at $\mathrm{x}=3 \mathrm{~mm}$ and $3.5 \mathrm{~mm}$. 


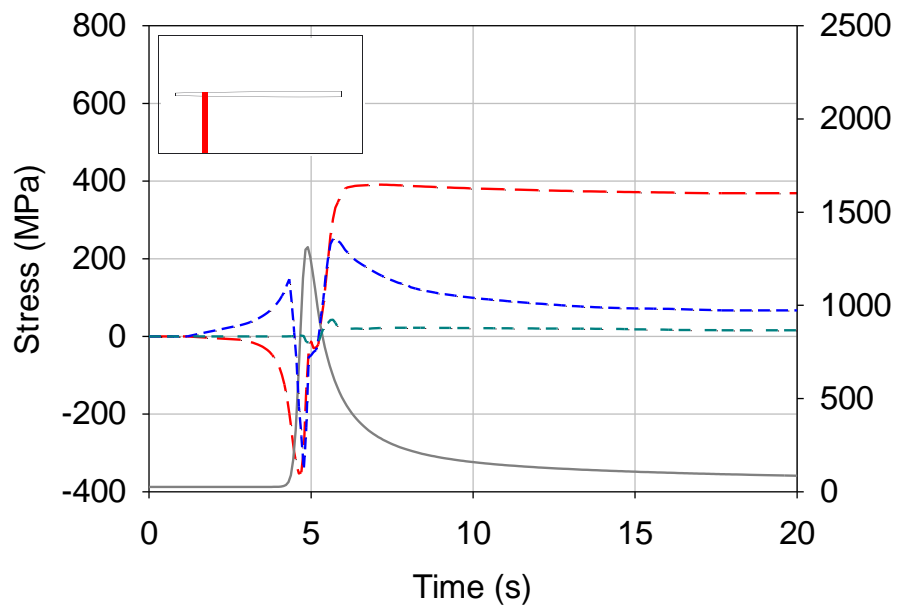

(a)

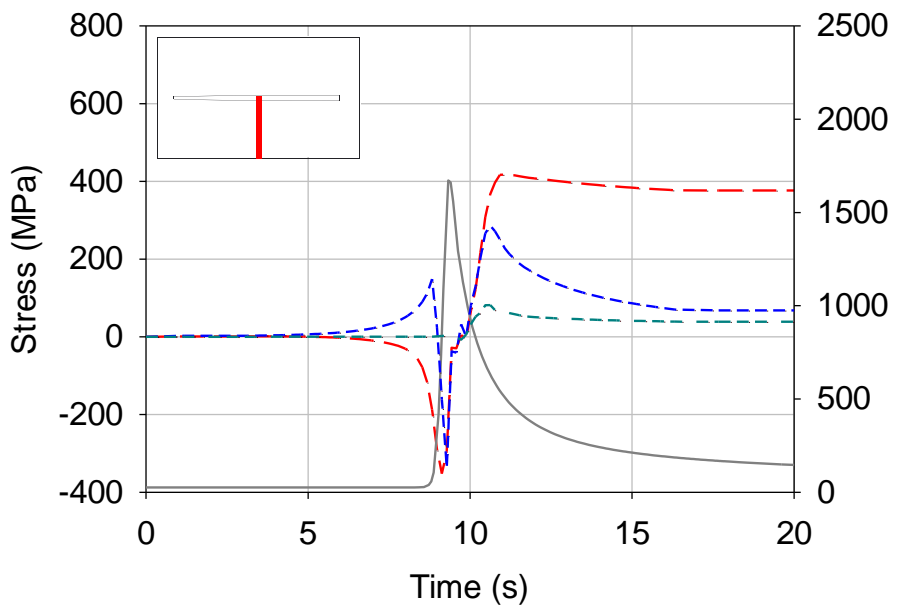

0
0
0
$\frac{0}{3}$
$\frac{\pi}{0}$
$\frac{0}{0}$
$\frac{0}{0}$
$\frac{1}{-}$

(b)

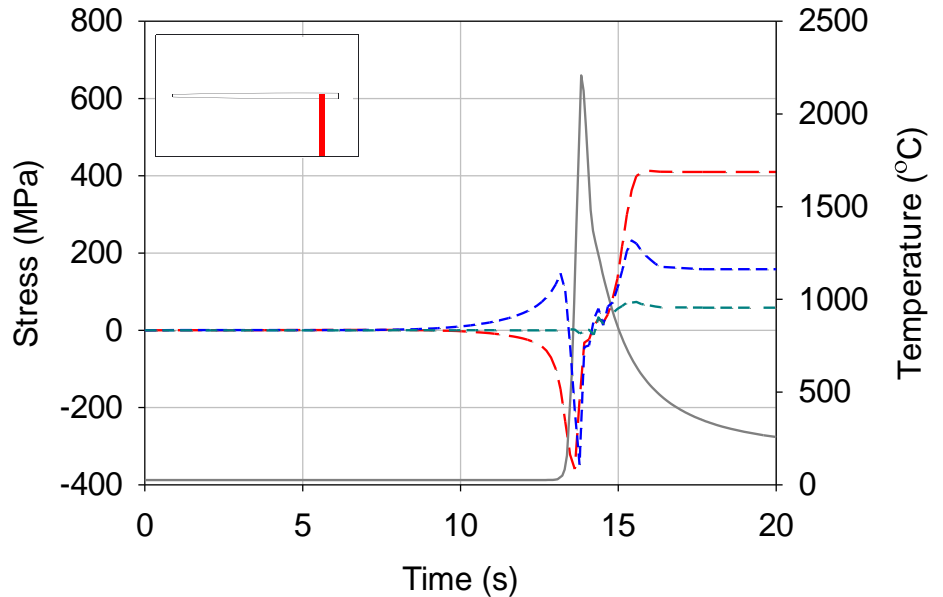

(c)

\begin{tabular}{llll}
------ & Longitudinal $\left(\sigma_{\mathrm{yy}}\right)$ & $-\cdots-\cdots-\cdots$ & Transverse $\left(\sigma_{\mathrm{xx}}\right)$ \\
\hline & Temperature & $-\cdots-\cdots--$ & Normal $\left(\sigma_{\mathrm{zz}}\right)$
\end{tabular}

Figure 20 Numerical prediction of the transverse, longitudinal and normal stress components during the deposition process, in three regions of the track (a) $y=30 \mathrm{~mm}$; (b) $y=60 \mathrm{~mm}$ and (c) $y=90 \mathrm{~mm}$. The nodal point was at the track centreline $0.25 \mathrm{~mm}$ below the substrate surface. 


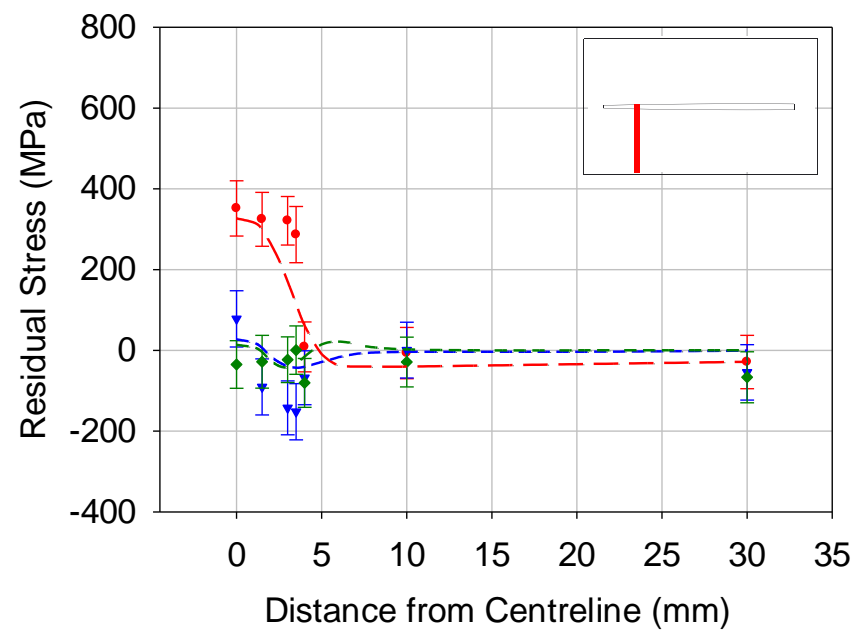

(a)

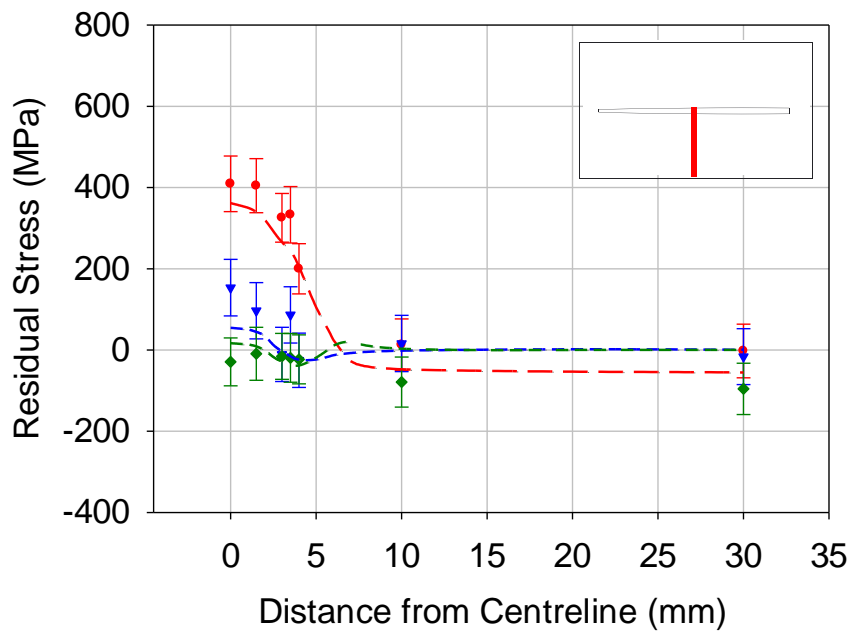

(b)

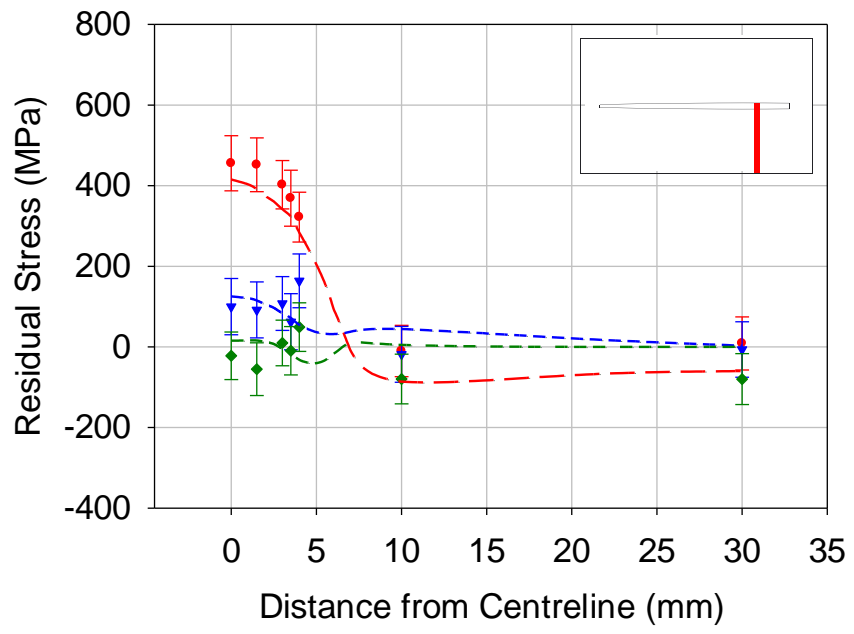

(c)

\begin{tabular}{|llll}
\hline & Longitudinal $\left(\sigma_{\mathrm{yy}}\right),(\mathrm{ND})$ & ------ & Longitudinal $\left(\sigma_{\mathrm{yyy}}\right),(\mathrm{FE})$ \\
& Transverse $\left(\sigma_{\mathrm{xx}}\right),(\mathrm{ND})$ & $-\cdots-\cdots$ & Transverse $\left(\sigma_{\mathrm{xx}}\right),(\mathrm{FE})$ \\
& Normal $\left(\sigma_{\mathrm{zz}}\right),(\mathrm{ND})$ & $-\cdots$ & Normal $\left(\sigma_{\mathrm{zz}}\right),(\mathrm{FE})$ \\
\hline
\end{tabular}

Figure 21 Comparison of experimentally measured and numerically predicted tri-axial residual stresses at $2 \mathrm{~mm}$ below the surface for each line scan position (a) y=30 mm; (b) y=60 $\mathrm{mm}$ and (c) $\mathrm{y}=90 \mathrm{~mm}$. 


\section{Discussion}

The work conducted in this study presents a novel modelling strategy, allowing in-situ process parameter control within DED to be modelled, by encompassing the key physics and parameters of the process. The modelling strategy can be extended to conduct parametric investigations to determine the effects of the process parameters on the resultant temperature and residual stress fields. Through the use of the modelling strategy presented here, tailored repair strategies can be devised and implemented for the repair of components utilising DED.

\subsection{Track Profile Prediction}

The evolution of the track profile was predicted using a numerical method and compared to focus variation microscopy measurements. A good agreement was attained between the predicted and experimental shape of the track profile at all of the locations. As the shape of the profile is governed by the powder distribution, an accurate representation of this variable has been achieved through the estimated Gaussian feed radius. The maximum error for the track profile shape was $7.9 \%$ this was due to the formation of an asymmetric bead profile. This was not captured by the model as the fluid flow upon solidification was not included. The variation in track height, through altering the laser power, has been captured well by the numerical prediction. Through increasing the laser power by a scale factor of 2 , the track height increased by $58.7 \%$; therefore, a directly proportional relationship is not observed. A maximum error of $6.5 \%$ was determined between the predicted an experimental track height. As the track height is predominantly governed by the powder catchment efficiency, the low error validates the method by which these parameters were derived. Therefore, the error in the predicted height could be because some powder particles may not reach the melt pool, as they could be melted in flight and/or be deflected from the substrate due to the high velocity carrier gas used in the experimental procedure. Overall, a higher percentage error was seen between the predicted and experimental track width. As this is governed by the maximum melt pool width, the assumption that the absorptivity was temperature independent may have caused this error as this would determine the peak heat flux entering the domain. Another potential reason may be due to the assumption that the Gaussian radius of the heat source was equal to the laser spot radius, as this would govern the distribution of thermal energy across the substrate and in turn would alter the geometry of the melt pool. For the track profile model, temperature independent material properties were used to implement the analytical expressions. The reason for this was to enable a more efficient solution to the analytical equations [8]; however, this simplification may cause the discrepancies observed between the predicted and experimental track profile. To assess the validity of this assumption, track profiles were derived for the steady-state regions, defining the density and specific heat capacity properties for temperatures greater than $630^{\circ} \mathrm{C}$. Across this range of material properties, the maximum error was associated with the track height 
was 1.4\%; therefore, using temperature independent properties has a negligible effect on the derived bead geometry, validating the assumption. It should be noted that the convective and radiative losses were neglected from this section of the model. Therefore, the predicted thermal energy within the domain is overestimated and in turn will result in an overestimate of the melt pool geometry. Therefore, this can lead to the discrepancies observed between the predicted and experimental track profiles. The overestimate in heat flux could also arise from the method in which the laser heat source is represented within the FE model. It was assumed for this work that the Gaussian radius was equal to half the size of the laser spot radius. This value was validated for the thermomechanical analysis; however, calibration of the heat source would have been more accurate without the deposition material present. It should also be noted that, from the substrate deforming, the laser spot size will change; therefore, it cannot be assumed that the Gaussian radius is constant throughout. As the melt pool width is also dependent upon the power loss history, derived from the mass-enthalpy balance, the analytical approximation of the power loss may provide an underestimate. This may result in the overestimate between the predicted and experimental size of the melt pool as observed in Figure 19.

\subsection{Thermal History and Melt Pool Profile}

The experimental and numerical predictions of the thermal histories correlate well with one another. The heating and cooling phases were captured by the FE model with accuracy, with slight discrepancies in the thermal gradient before the heat source pass. An overestimate in the peak temperature values for all thermocouples was observed, this is due to the method in which ABAQUS applies the heat source model to the FE domain [32]. It appears that the cooling rate at each horizontal location changes, which may arise due to the in-situ deformation of the substrate, reducing the contact area between the substrate and the CNC bed. As this heat loss was modelled using an artificial, convective boundary condition, this could not be accounted for. To model the CNC bed within the FE domain would increase the number of elements within the mesh, therefore reducing the computational efficiency of the simulation. It should be noted that the thermocouple was mounted to the substrate manually and from the FE model, a $0.2 \mathrm{~mm}$ position change gave rise to a $9 \%$ difference in the temperature.

To further validate the FE heat source, a comparison of the predicted fusion zone and the one derived from the experimental micrograph was conducted. The overall predicted shape of the fusion zone agreed well with the experimental micrograph, with an increase in penetration depth observed with an increase in laser power. The width of the fusion zone was predicted accurately; however, the predicted penetration depth was underestimated for the low power and transient regions. A possible reason for why this inaccuracy is present within the model maybe due to the melt pool and track width not being coherent. This could be due to an overestimate in the predicted track geometry for the aforementioned reasons. 
Another cause maybe due to the assumption that the absorptivity is constant and assumed to be temperature independent throughout the analysis. .

\subsection{Residual Stress Predictions}

Through the use of ND measurements, the mechanical aspects of the FE model were validated. Overall, the residual stress profiles for the experimental and predicted data sets correlated well for all three stress components at each horizontal line scan. At each line scan location, the trends of the triaxial residual stresses have been captured well by the FE model. Almost all of the predicted residual stress values fall within the confidence limits of the experimental measurements; however, the greatest errors occur within the vicinity of the track. Limited variation was present during the evolution of stress within the melt pool region and in the final residual stress state of the component. The transverse stress component differs at each horizontal location, with the stress direction at $\mathrm{y}=90 \mathrm{~mm}$ being entirely tensile, as the increased cooling rate in this region does not allow a redistribution of stress to occur. At $y=30 \mathrm{~mm}$ and $60 \mathrm{~mm}$ a sharp stress gradient is present in the vicinity of the track, as the reduced cooling rate allows the track material to contract and exhibit a compressive stress at the substrate surface. At each horizontal line scan location, an increase in magnitude of the longitudinal stress component was observed, with this being captured well by the FE model; however, a greater increase was determined from the experimental measurements. This may have arisen due to the underestimate in the penetration depth from the thermal FE model, resulting in a smaller volume of material contracting upon cooling. Another potential reason for this error may be due to neglecting the restraining bolts from the FE model, as this would alter the evolution of stress within the substrate. Including this effect within the FE model would inherently increase its complexity and simulation time. Other reasons for the discrepancies observed between residual stresses obtained from the ND measurements and FE model could include; experimental positioning errors, microstructure evolution within the substrate and the variation in measurement temperature. To ensure that a reliable stress measurement is derived from the ND measurements, the location at which the ND measurement is taken in the region of interest and within the strain free reference sample has to be identical. As these measurements were not taken utilising the same datum reference, a positioning error could be introduced, leading to an incoherent comparison between the two data sets. At the location of $y=30 \mathrm{~mm}$ and $\mathrm{y}=90 \mathrm{~mm}$, a positioning error at $\mathrm{x}=3$ and $3.5 \mathrm{~mm}$ is believed to be the reason for the large difference observed between the predicted and experimental residual stress. Minimising positioning errors will ensure that the gauge volume is completely filled, neglecting the measurement of pseudo strain, giving rise to the error in the direction of the measured and predicted stress at these locations. Also, as the substrate will deform during the deposition process, the camber and butterfly distortion should be considered and accounted for whilst 
creating the datum for the ND measurements. This was not completed for this experiment and could have led to the error observed between the two data sets.

Due to the evolution of the track geometry and the variation in laser power, microstructure variation will be present at each horizontal line scan. The strain free reference sample was extracted from the location where $\mathrm{P}=1800 \mathrm{~W}$. In this region, the highest peak melt pool temperature was present $\left(2598^{\circ} \mathrm{C}\right)$, allowing sufficient time for the diffusion of the strengthening precipitates, $\gamma^{\prime \prime}(\mathrm{Ni} 3 \mathrm{Nb})$ and $\gamma^{\prime \prime}\left(\mathrm{Ni}_{3} \mathrm{Al}\right)$ [43], to take place. This occurs due to the peak temperature in the melt pool exceeding the optimum temperature $\left(1150^{\circ} \mathrm{C}\right)[44]$ for the precipitates to enter solution, therefore resulting in crystallographic mismatch. This, in turn, gives rise to varying magnitudes of intergranular strain in the vicinity of the Heat Effects Zone (HAZ), at the position of each horizontal line scan. From analysing the location where the thermal field equated the recrystallisation temperature $\left(1020^{\circ} \mathrm{C}\right)$ of IN718 [43], the approximate width and depth of the HAZ was $1.6 \times 0.75 \mathrm{~mm}^{2}$ and $2 \times 1.25 \mathrm{~mm}^{2}$ for $\mathrm{P}=900 \mathrm{~W}$ and $\mathrm{P}=1800 \mathrm{~W}$ respectively (Figure 22). In the high-power region, the gauge volume included a greater volume of the HAZ compared to the low-power region, leading to an incoherent comparison between the strain-free lattice spacing and the region of interest. This is true for the horizontal line scan at $y=30 \mathrm{~mm}$ and $y=60 \mathrm{~mm}$, as the variation of intergranular strain is not accounted for utilising one do specimen. As the macrostress is of interest, a shift in this variable will be present, as the difference between the lattice spacing before cutting of the $d_{0}$ specimen, and the reference spacing measured for the same region was no longer proportional to the macrostress [40]. Therefore, to reduce the error obtained in the experimental residual stress profiles, a strain free reference specimen should be utilised at each horizontal line scan location. Finally, the ND measurements were not completed at constant ambient conditions and would cause thermally induced intergranular strains, due to anisotropic thermal expansion [40]. As the measurements were completed over a 24 hour period, a significant change in lattice spacing would therefore occur. 


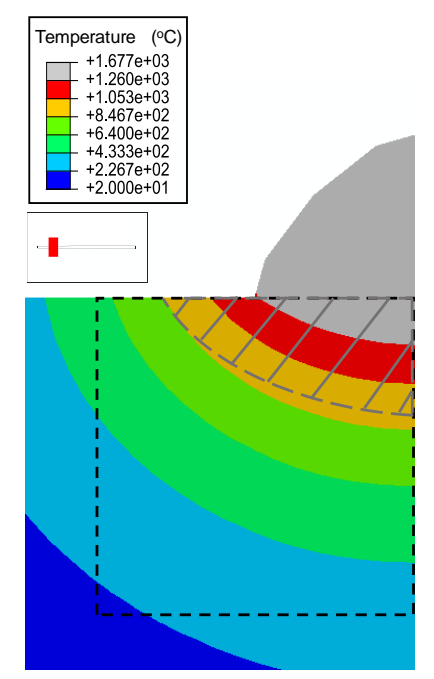

(a)

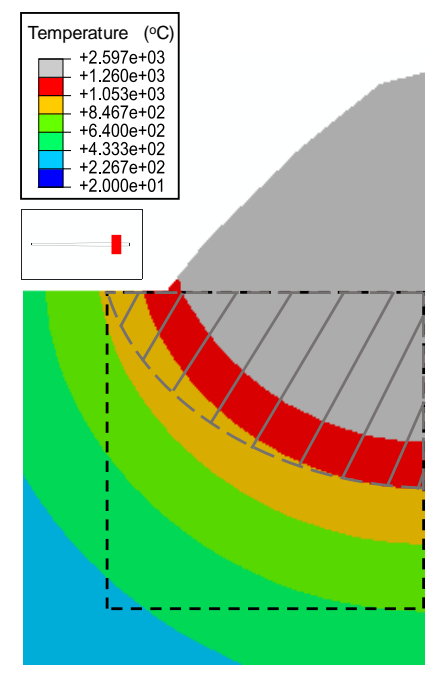

(b)

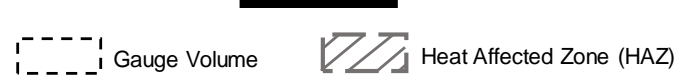

Figure 22 Schematic of the estimated $\mathrm{HAZ}$ and the ND gauge volume for (a) $\mathrm{y}=30 \mathrm{~mm}$ and (b) $y=90 \mathrm{~mm}$.

From this work, it is confirmed that high magnitude residual stresses are present within DED manufactured components; therefore, methods to control the evolution of these stresses in DED parts will need more attention in order to extend the service life of components repaired using this technology. From this study, a variation in the laser power does not considerably alter the evolution of residual stresses as there is minimal variation in the trend and magnitude at each line scan location. As the cooling rates for the process are high, it is believed that controlling this phenomenon before or after the deposition would help to reduce high magnitude residual stress formation. However, the application of this model should be extended to multi-layer deposition, to determine if in-situ parameter variation has a substantial effect on the thermomechanical behaviour of the repaired component. As the current study presents a generalised model to predict the effect of adaptive process control, the modelling approach can be extended for multi-track deposition. In general, the current approach can be applied until the melt pool geometry has to be determined. Due to the overlap between the melt pool and the previously deposited track, the geometry can no longer be represented as two elliptical sections. Methods exist to model overlapping melt pool geometries, such as an analytical representation presented by Ahsan et al. [45]; however, the inclusion of this new phenomena is not trivial and the validity of the approach has to be evaluated. Therefore, further work to extend the current approach to multi-deposition is required.

The current modelling methodology presented will aid as a design tool to perform multi combination parametric studies of the processing parameters. The track profile and thermomechanical effects can then be evaluated to ensure that the optimum repair strategy is implemented for the component. The modelling strategy will therefore allow a bespoke 
repair application to be adopted which can restore the damaged component to its original in-service condition.

\section{Conclusions}

A new method is presented which encompasses the manufacturing flexibility of the DED process into a single modelling strategy. The modelling method allows a realistic, 3D transient track profile to be predicted and then implemented into a FE model to evaluate the thermal and mechanical effects of the process. The approach allows multiple process parameters to be investigated, allowing a tailored repair strategy to be identified. The key findings of this study are presented below.

- The modelling strategy presented here can aid in devising an optimum set of process parameters to ensure a geometrically accurate build is attained and to assess the combination of process parameters which can control the cooling rate and the evolution of stress within the component. However, as the validity of the approach is for a single track, the modelling strategy will be extended to evaluate multi track depositions.

- The validated case presented in this study agrees well with the experimental data showing confidence in the modelling strategy. The maximum track height and width were predicted with a maximum error of $6.5 \%$ and $7.62 \%$ respectively. The peak temperatures and residual stresses were underestimated with an error of $6.2 \%$ and $11.4 \%$ respectively.

- Minimal variation was observed in the trend for the stress evolution and the final residual stress state at each horizontal location. The longitudinal stress increased with laser power, with negligible deviation in the other two stress components. A plane stress condition was not observed due to the interaction of the track geometry and the substrate at the interface between them.

- From this study, total mesh independence can only be achieved when evaluating both the accuracy of the thermal history and melt pool geometry. To ensure the optimum element size is utilised to represent the heat flux for the selected process parameters, a method to calibrate the FE heat source is required to reduce the error in the numerically derived results.

- High magnitude residual stresses are present in a single-track deposition with a minimal reduction being observed through controlling the laser power. As these types of stresses will reduce the life of the component, further study should be conducted to determine methods that adaptively control the evolution of high magnitude stresses. 


\section{Acknowledgement}

We are grateful for access to the University of Nottingham's Augusta HPC service.

\section{References}

[1] P. Peyre, P. Aubry, R. Fabbro, R. Neveu, and A. Longuet, "Analytical and numerical modelling of the direct metal deposition laser process," J. Phys. D. Appl. Phys., vol. 41, no. 2, p. 025403, 2008 doi:10.1088/0022-3727/41/2/025403.

[2] S. M. Thompson, L. Bian, N. Shamsaei, and A. Yadollahi, "An overview of Direct Laser Deposition for additive manufacturing; Part I: Transport phenomena, modeling and diagnostics," Additive Manufacturing, vol. 8. Elsevier B.V., pp. 36-62, 2015 doi:10.1016/j.addma.2015.07.001.

[3] A. J. Pinkerton, "Advances in the modeling of laser direct metal deposition," J. Laser Appl., vol. 27, no. S1, p. S15001, 2014 doi:10.2351/1.4815992.

[4] S. Kumar, V. Sharma, A. K. S. Choudhary, S. Chattopadhyaya, and S. Hloch, "Determination of layer thickness in direct metal deposition using dimensional analysis," Int. J. Adv. Manuf. Technol., vol. 67, no. 9-12, pp. 2681-2687, 2013 doi:10.1007/s00170-012-4683-1.

[5] J. Paulo Davim, C. Oliveira, and A. Cardoso, "Predicting the geometric form of clad in laser cladding by powder using multiple regression analysis (MRA)," Mater. Des., vol. 29, no. 2, pp. 554-557, 2008 doi:10.1016/j.matdes.2007.01.023.

[6] M. Picasso, C. F. Marsden, J. D. Wagniere, A. Frenk, and M. Rappaz, "A simple but realistic model for laser cladding," Metall. Mater. Trans. B, vol. 25, no. 2, pp. 281-291, 1994 doi:10.1007/BF02665211.

[7] A. J. Pinkerton and L. Li, "Modelling the geometry of a moving laser melt pool and deposition track via energy and mass balances," J. Phys. D. Appl. Phys., vol. 37, no. 14, pp. 1885-1895, 2004 doi:10.1088/0022-3727/37/14/003.

[8] M. N. Ahsan and A. J. Pinkerton, "An analytical-numerical model of laser direct metal deposition track and microstructure formation," Model. Simul. Mater. Sci. Eng., vol. 19, no. 5, p. 055003, Jul. 2011 doi:10.1088/0965-0393/19/5/055003.

[9] N. Tamanna, R. Crouch, I. R. Kabir, and S. Naher, "An analytical model to predict and minimize the residual stress of laser cladding process," Appl. Phys. A Mater. Sci. Process., vol. 124, no. 2, pp. 1-5, 2018 doi:10.1007/s00339-018-1585-6.

[10] P. Michaleris, "Modeling metal deposition in heat transfer analyses of additive manufacturing processes," Finite Elem. Anal. Des., vol. 86, pp. 51-60, 2014 doi:10.1016/j.finel.2014.04.003.

[11] J. Lin, "Numerical simulation of the focused powder streams in coaxial laser cladding," $J$. Mater. Process. Technol., vol. 105, no. 1, pp. 17-23, 2000 doi:10.1016/S09240136(00)00584-7.

[12] J. Ibarra-Medina, M. Vogel, and A. J. Pinkerton, "A CFD model of laser cladding: From deposition head to melt pool dynamics," in 30th International Congress on Applications of Lasers and Electro-Optics, ICALEO 2011, 2018, pp. 378-386 doi:10.2351/1.5062261.

[13] M. Labudovic and R. Kovacevic, "A three dimensional model for direct laser metal," J. Mater. Sci., vol. 38, pp. 35-49, 2003 doi:10.1002/jcop.20152.

[14] P. Farahmand and R. Kovacevic, "An experimental-numerical investigation of heat distribution and stress field in single- and multi-track laser cladding by a high-power direct diode laser," Opt. Laser Technol., vol. 63, pp. 154-168, 2014 doi:10.1016/j.optlastec.2014.04.016.

[15] Z. Zhang and R. Kovacevic, "A thermo-mechanical model for simulating the temperature and stress distribution during laser cladding process," Int. J. Adv. Manuf. Technol., vol. 102, no. 14, pp. 457-472, 2019 doi:10.1007/s00170-018-3127-y.

[16] T. R. Walker, C. J. Bennett, T. L. Lee, and A. T. Clare, "A validated analytical-numerical modelling strategy to predict residual stresses in single-track laser deposited IN718," Int. J. Mech. Sci., vol. 151, pp. 609-621, Feb. 2019 doi:10.1016/j.ijmecsci.2018.12.004.

[17] M. Alimardani, E. Toyserkani, and J. P. Huissoon, "Three-dimensional numerical approach for geometrical prediction of multilayer laser solid freeform fabrication process," J. Laser Appl., vol. 19, no. 1, pp. 14-25, 2006 doi:10.2351/1.2402518.

[18] V. Pavelic, R. Tanbakuchi, O. . Uyehara, and P. . Myers, "Welding Journal Research Supplement," vol. 48, pp. 295-305, 1969.

[19] A. J. Pinkerton and L. Li, "Modelling Powder Concentration Distribution From a Coaxial Deposition Nozzle for Laser-Based Rapid Tooling," J. Manuf. Sci. Eng., vol. 126, no. 1, p. 33, 2004 doi:10.1115/1.1643748.

[20] K. Partes, "Analytical model of the catchment efficiency in high speed laser cladding," Surf. Coatings Technol., vol. 204, no. 3, pp. 366-371, 2009 doi:10.1016/j.surfcoat.2009.07.041. 
[21] A. J. Pinkerton, R. Moat, K. Shah, L. Li, M. Preuss, and P. J. Withers, "A verified model of laser direct metal deposition using an analytical enthalpy balance method," in Proceedings of the 26th International Congress on Applications of Lasers and Electro-optics (ICALEO), 2018, p. 1806 doi:10.2351/1.5061038.

[22] M. Salcudean, M. Choi, and R. Greif, "A study of heat transfer during arc welding," Int. J. Heat Mass Transf., vol. 29, no. 2, pp. 215-225, Feb. 1986 doi:10.1016/0017-9310(86)90229-2.

[23] A. Lundbäck and L. E. Lindgren, "Modelling of metal deposition," Finite Elem. Anal. Des., vol. 47, no. 10, pp. 1169-1177, 2011 doi:10.1016/j.finel.2011.05.005.

[24] K. Dai and L. Shaw, "Thermal and stress modeling of multi-material laser processing," Acta Mater., vol. 49, no. 20, pp. 4171-4181, 2001 doi:10.1016/S1359-6454(01)00312-3.

[25] ABAQUS Analysis User's Manual Version 6.13. USA: Dassault Systèmes Simulia Corp, 2013.

[26] T. E. Abioye, J. Folkes, and A. T. Clare, "A parametric study of Inconel 625 wire laser deposition," J. Mater. Process. Technol., vol. 213, no. 12, pp. 2145-2151, 2013 doi:10.1016/j.jmatprotec.2013.06.007.

[27] X. Liang, L. Cheng, Q. Chen, Q. Yang, and A. C. To, "A modified method for estimating inherent strains from detailed process simulation for fast residual distortion prediction of singlewalled structures fabricated by directed energy deposition," Addit. Manuf., vol. 23, no. August, pp. 471-486, 2018 doi:10.1016/j.addma.2018.08.029.

[28] D. Dye, O. Hunziker, S. M. Roberts, and R. C. Reed, "Modeling of the mechanical effects induced by the tungsten inert-gas welding of the IN718 superalloy," Metall. Mater. Trans. A Phys. Metall. Mater. Sci., vol. 32, no. 7, pp. 1713-1725, 2001 doi:10.1007/s11661-001-0149-z.

[29] J. J. Valencia and Quested P.N, "Thermophysical Properties," ASM Handb. Cast., vol. 15, no. Ref 24, pp. 468-481, 2008 doi:10.1361/asmhba0005240.

[30] C. L. Chan and J. Mazumder, "One-dimensional steady-state model for damage by vaporization and liquid expulsion due to laser-material interaction," J. Appl. Phys., vol. 62, no. 11, pp. 4579-4586, 1987 doi:10.1063/1.339053.

[31] M. Anderson, R. Patwa, and Y. C. Shin, "Laser-assisted machining of Inconel 718 with an economic analysis," Int. J. Mach. Tools Manuf., vol. 46, no. 14, pp. 1879-1891, 2006 doi:10.1016/j.ijmachtools.2005.11.005.

[32] A. A. Deshpande, D. W. J. Tanner, W. Sun, T. H. Hyde, and G. McCartney, "Combined Butt Joint Welding and Post Weld Heat Treatment Simulation Using SYSWELD and ABAQUS," Proc. Inst. Mech. Eng. Part L J. Mater. Des. Appl., vol. 225, no. 1, pp. 1-10, Jan. 2011

doi:10.1177/14644207JMDA349.

[33] E. R. Denlinger, J. C. Heigel, P. Michaleris, and T. A. Palmer, "Effect of inter-layer dwell time on distortion and residual stress in additive manufacturing of titanium and nickel alloys," $J$.

Mater. Process. Technol., vol. 215, pp. 123-131, 2015 doi:10.1016/j.jmatprotec.2014.07.030.

[34] H. Yoshimura and K. Winterton, "Solidification Mode of Weld Metal in Inconel 718," Weld. J. Res. Suppl., vol. 51, pp. 132-137, 1972.

[35] E. R. Denlinger and P. Michaleris, "Effect of stress relaxation on distortion in additive manufacturing process modeling," Addit. Manuf., vol. 12, pp. 51-59, 2016 doi:10.1016/j.addma.2016.06.011.

[36] O. Muránsky, C. J. Hamelin, M. C. Smith, P. J. Bendeich, and L. Edwards, "The effect of plasticity theory on predicted residual stress fields in numerical weld analyses," Comput. Mater. Sci., vol. 54, no. 1, pp. 125-134, 2012 doi:10.1016/j.commatsci.2011.10.026.

[37] G. Salerno et al., "On the interaction between welding residual stresses: a numerical and experimental investigation $0 n$ the interaction between welding residual stresses: a numerical and experimental investigation," Int. J. Mech. Sci., vol. 144, no. 17, pp. 654-667, 2018 doi:10.1016/j.ijmecsci.2018.04.055.

[38] P. Foteinopoulos, A. Papacharalampopoulos, and P. Stavropoulos, "On thermal modeling of Additive Manufacturing processes,” CIRP J. Manuf. Sci. Technol., vol. 20, pp. 66-83, 2018 doi:10.1016/j.cirpj.2017.09.007.

[39] Oerlikon, "Oerlikon AM: Additive Manufacturing 718 Nickel Alloy Data Sheet."

[40] P. J. Withers, M. Preuss, A. Steuwer, and J. W. L. Pang, "Methods for obtaining the strain-free lattice parameter when using diffraction to determine residual stress," J. Appl. Crystallogr., vol. 40, no. 5, pp. 891-904, 2007 doi:10.1107/S0021889807030269.

[41] J. R. Santisteban, M. R. Daymond, J. A. James, and L. Edwards, "ENGIN-X: A third-generation neutron strain scanner," J. Appl. Crystallogr., vol. 39, no. 6, pp. 812-825, 2006 doi:10.1107/S0021889806042245.

[42] D. Dye, S. M. Roberts, P. J. Withers, and R. C. Reed, "Determination of the residual strains and stresses in a tungsten inert gas welded sheet of IN718 superalloy using neutron diffraction," J. Strain Anal. Eng. Des., vol. 35, no. 4, pp. 247-259, 2000 doi:10.1243/0309324001514396.

[43] H. Y. Zhang, S. H. Zhang, M. Cheng, and Z. Zhao, "Microstructure evolution of IN718 alloy during the delta process," in Procedia Engineering, 2017, vol. 207, pp. 1099-1104 doi:10.1016/j.proeng.2017.10.1137.

[44] X. You, Y. Tan, S. Shi, J. Yang, Y. Wang, and J. Li, "Effect of solution heat treatment on the precipitation behavior and strengthening mechanisms of electron beam smelted Inconel 718 superalloy," Mater. Sci. Eng. A, vol. 689, no. 2, pp. 257-268, 2017 
doi:10.1016/j.msea.2017.01.093.

[45] M. N. Ahsan, C. P. Paul, L. M. Kukreja, and A. J. Pinkerton, "Porous structures fabrication by continuous and pulsed laser metal deposition for biomedical applications; Modelling and experimental investigation," J. Mater. Process. Technol., vol. 211, no. 4, pp. 602-609, 2011 doi:10.1016/j.jmatprotec.2010.11.014. 